\title{
Relative Algebraic Reduction and Relative Albanese Map for a Fiber Space in $\mathcal{C}$
}

By

\section{Akira FuJIKI*}

\section{Introduction}

Let $f: X \rightarrow Y$ be a fiber space of compact complex manifolds, i. e., $f$ is surjective with connected fibers. Let $U \subseteq Y$ be a Zariski open subset over which $f$ is smooth. Then for each $y \in U$ we have the Albanese map $\phi_{y}: X_{y} \rightarrow \operatorname{Alb} X_{y}$ of $X_{y}:=f^{-1}(y)$. Under a suitable condition, e. g., if $X_{y}$ is a manifold in $\mathcal{C}$ (i. e., $X_{y}$ is a meromorphic image of a compact Kähler manifold), then the collection $\left\{\right.$ Alb $X_{y}$ \} can be put together to form a complex manifold Alb $X_{U} / U$ over $U$ and $\left\{\psi_{y}\right\}$ to form a holomorphic map $\psi_{U}: X_{U} \rightarrow$ Alb $X_{U} / U$ over $U$ where $X_{U}=f^{-1}(U)$. Then the main problem to be treated in this paper is the following: When can we compactify Alb $X_{U} / U$ to a compact complex manifold Alb* $X / Y$ over $Y$ so that $\psi_{U}$ extends to a meromorphic map $\phi: X \rightarrow \mathrm{Alb}^{*} X / Y$ over $Y$ ? (Here we do not require any good property for $\mathrm{Alb} * X / Y$; any compactification is enough for our purpose.) We shall show in this paper that this is the case if i) the total space $X$ is in $\mathcal{C}$, and ii) any smooth fiber $X_{y}$ is Moishezon, (after a possible restriction of $U$ ). Moreover it turns out that in this case $\mathrm{Alb}^{*} X / Y$ is again in $\mathcal{C}$ and the pair $\left(\phi, \mathrm{Alb}^{*} X / Y\right)$ is unique up to bimeromorphic equivalences. We call $\phi$ briefly the relative Albanese map for $f$. One notable property of $\psi$ we prove is that it is Moishezon in the sense that it is bimeromorphic to a projective morphism. Thus, in a sense, the relative Albanese variety $\mathrm{Alb}^{*} X / Y$ may be considered as the obstruction for a fiber space with general fiber Moishezon to be a Moishezon morphism.

We follow the method of Grothendieck [13] in algebraic geometry, constructing Alb $X_{U} / U$ as a component of the relative Picard variety $\mathrm{Pic}\left(\left(\mathrm{Pic}_{\gamma} X_{U} / U\right) / U\right)$ of some component $\mathrm{Pic}_{r} X_{U} / U$ of the relative Picard variety $\mathrm{Pic} X_{U} / U$ of $X_{U}$ over $U$. Here Pic $X_{U} / U$ (or at least a good part of it) in turn is constructed as a flat quotient of the space $\operatorname{Div} X_{U} / U$ of relative divisors on $X_{U}$ over $U$.

Our first step is thus to construct a natural completion $\operatorname{Div} * X / Y$ of $\operatorname{Div} X_{U} / U$ over $Y$, where the assumption that $X \in \mathcal{C}$ is essential to guarantee that each irreducible component of $\operatorname{Div}^{*} X / Y$ is compact (Section 2). The second step is then to complete Pic $X_{U} / U$ to a complex variety Pic* $X / Y$ over $Y$ such that the

Communicated by S. Nakano, April 5, 1982.

* Yoshida College, Kyoto University, Kyoto 606, Japan. 
natural morphism $\mu_{X_{U} / U}: \operatorname{Div} X_{U} / U \rightarrow \operatorname{Pic} X_{U} / U$ extends to a meromorphic map $\mu_{X / Y}^{*}: \operatorname{Div}^{*} X / Y \rightarrow \operatorname{Pic}^{*} X / Y$ (Section 3 ). This will be done through a simple but useful lemma (Lemma 9). Here, however, for the method of [13] to be applicable it is necessary to show that after passing to another bimeromorphic model of $X$ (which is admissible because of the bimeromorphic invariance of Albanese map) any general fiber of $f$ becomes projective. This is also done in Section 2. The final step is the construction of $\mathrm{Alb}^{*} X / Y$ from $\mathrm{Pic}^{*} X / Y$ and will be given in Section 4.

Though it is expected that the second condition of $X_{y}$ being Moishezon is irrelevant for the existence theorem, our method gives no idea for the general case.

In Section 2, in relation with our study of the space $\operatorname{Div}^{*} X / Y$ we also develop the theory of relative algebraic reduction, i. e., we show that for any fiber space $f: X \rightarrow Y$ in $\mathcal{C}$ we can always construct a compact complex manifold $Z$ over $Y$ and surjective meromorphic map $g: X \rightarrow Z$ such that for 'general' $y \in Y g$ induces a meromorphic map $g_{y}: X_{y} \rightarrow Z_{y}$ which is an algebraic reduction of $X_{y}$. We note that this theory of relative algebraic reduction has also been developped by Campana [3] independently. Both relative Albanese maps and relative algebraic reductions provide us with fundamental tools for our investigation of the structure of compact complex manifolds in $\mathcal{C}$ in [11], which was actually the motivation for this paper. The results in this paper were announced in [8] and $[8 \mathrm{a}]$.

Notations and Convention. A complex variety means a reduced and irreducible complex space. As above a fiber space is a proper surjective holomorphic map with general fiber irreducible. A compact complex space $X$ is said to be in the class $\mathcal{C}$ if $X_{\text {red, }}$, the underlying reduced subspace of $X$, is a meromorphic image of a compact Kähler manifold (cf. [5]). (Notation $X \in \mathcal{C}$ ) A Zariski open subset of a complex variety is always assumed to be nonempty. Let $f: X \rightarrow Y$ be a morphism of complex space. Then for any morphism $\tilde{Y} \rightarrow Y$ we often write $X_{\tilde{Y}}=X \times_{Y} \tilde{Y}$ and $f_{\tilde{Y}}:=f \times_{Y} i d_{\tilde{Y}}: X_{\tilde{Y}} \rightarrow \tilde{Y}$. Let $f^{\prime}: X^{\prime} \rightarrow Y$ be another complex space and $g: X \rightarrow X^{\prime}$ a meromorphic map over $Y$. Then for any open subset $U \subseteq Y$ we often denote by $g_{U}$ the restriction of $g$ to $X_{U} ; g_{U}=\left.g\right|_{X_{U}}: X_{U} \rightarrow X_{U}^{\prime}$.

Let $f: X \rightarrow Y$ and $f^{\prime}: X^{\prime} \rightarrow Y$ be morphisms of compact reduced complex spaces. Suppose that $Y$ is a variety and any irreducible component of $X$ and $X^{\prime}$ is mapped surjectively onto $Y$. Let $U \subseteq Y$ be a Zariski open subset over which $f$ is flat. Let $\tilde{U}=f^{\prime-1}(U)$. Then the closure of $X \times_{U} \tilde{U}$ in $X \times_{Y} X^{\prime}$ is analytic and is independent of the choice of $U$ as above. Then we call this closure the strict pull-back of $X$ by $f^{\prime}$ and denote it by $X \dot{x}_{Y} X^{\prime}$. Then it is readily verified that $X \dot{\times}_{Y} X^{\prime} \cong X^{\prime} \dot{\times}_{Y} X$ with respect to the natural isomorphism $X \times_{Y} X^{\prime}$ $\cong X^{\prime} \times_{Y} X$ so that the formation of $X \dot{\times}_{Y} X^{\prime}$ is symmetric in $X$ and $X^{\prime}$. We denote the induced morphisms $X \dot{\mathrm{X}}_{Y} X^{\prime} \rightarrow X^{\prime}$ and $X \dot{\times}_{Y} X^{\prime} \rightarrow Y$ by $f \dot{\times}_{Y} X^{\prime}$ and $f \dot{X}_{Y} f^{\prime}$ respectively. We note that the above definition extends naturally to 
those $X$ and $X^{\prime}$ which are unions of compact complex varieties satisfying the above conditions.

\section{$\S 1$. Preliminaries}

In this section, mainly to fix notations, we shall review the generalities on relative Picard varieties $\operatorname{Pic} X / Y$, the space of relative divisors $\operatorname{Div} X / Y$, and the relative Albanese map Alb $X / Y$, for a proper smooth morphism $f: X \rightarrow Y$ (1.1-1.4); we also introduce the notion of s. ampleness of a line bundle and give a certain description of Albanese map in the absolute case. We denote by (An/ $/ Y^{\prime}$ ) the category of complex spaces over $Y$.

1.1. Pic $X / Y$. Let $f: X \rightarrow Y$ be a proper smooth morphism of complex varieties.

a) Define a contravariant functor Pic $X / Y:(\mathrm{An} / Y) \rightarrow($ Sets $)$ by Pic $X / Y\left(Y^{\prime}\right)$ : $=\Gamma\left(Y^{\prime}, R^{1} f_{Y^{\prime}} \mathcal{O}_{X \times Y^{\prime}}^{*}\right)$ where $f_{Y^{\prime}}=f \times_{Y^{\prime}} i d_{Y^{\prime}}: X \times_{Y} Y^{\prime} \rightarrow Y^{\prime}$. Then Pic $X / Y$ is represented by a commutative complex Lie group Pic $X / Y$ over $Y$. (See Bingener [2], and when $f$ is locally projective, Grothendieck [14].)

We denote by $b=b_{X / Y}$ : Pic $X / Y \rightarrow Y$ the structural morphism, and write $m=m_{X / Y}:$ Pic $X / Y \times_{Y}$ Pic $X / Y \rightarrow$ Pic $X / Y$ for the relative group multiplication and $\iota_{X / Y}:$ Pic $X / Y \rightarrow \operatorname{Pic} X / Y$ for the relative group inversion as a complex Lie group over $Y$. (For relative complex Lie group over $Y$, see [10] or [20].) Then we set $a=a_{X / Y}: \operatorname{Pic} X / Y \times_{Y} \operatorname{Pic} X / Y \rightarrow \operatorname{Pic} X / Y, a=m\left(i d_{\mathrm{Pic} X / Y} \times_{Y^{\ell} X / Y}\right)$ (the relative subtraction). When $Y$ is a point, we write $\operatorname{Pic} X$ for $\operatorname{Pic} X / Y$. We have then the natural isomorphism $\operatorname{Pic} X \cong H^{1}\left(X, \mathcal{O}_{X}^{*}\right)$.

b) Functorial properties of Pic $X / Y$. i) For any complex space $\tilde{Y}$ over $Y$ we have the natural isomorphism $P: \operatorname{Pic}\left(X \times_{Y} \tilde{Y} / \tilde{Y}\right) \cong \operatorname{Pic} X / Y \times_{Y} \tilde{Y}$. In particular for any $y \in Y$, $(\operatorname{Pic} X / Y)_{y}$ is naturally identified with Pic $X_{y}$ so that each point $p \in \operatorname{Pic} X / Y$ represents a unique line bundle $L_{p}$ on $X_{b(p)}$. ii) Let $f^{\prime}: X^{\prime} \rightarrow Y$ be another proper smooth morphism and $g: X^{\prime} \rightarrow X$ a $Y$-morphism. Then $g$ induces a natural $Y$-homomorphism $g^{*}: \operatorname{Pic} X / Y \rightarrow \operatorname{Pic} X^{\prime} / Y$.

c) By the definition of $\operatorname{Pic} X / Y$ there exists a universal section $l \in \Gamma\left(\operatorname{Pic} X / Y, R^{1} f_{\operatorname{Pic} X / Y{ }^{*}} \mathcal{O}_{X \times Y}^{*} \operatorname{Pic} X / Y\right)$ where $f_{\operatorname{Pic} X / Y}:=f \times_{Y} i d_{\operatorname{Pic} X / Y}: X \times_{Y} \operatorname{Pic} X / Y$ $\rightarrow$ Pic $X / Y$. In particular for any complex space $\tilde{Y}$ over $Y$ and an invertible sheaf $\mathcal{L}$ on $X \times_{Y} \tilde{Y}$ there exists a unique $Y$-morphism $\tau: \tilde{Y} \rightarrow \operatorname{Pic} X / Y$ such that the pull-back of $l$ by $\tau$ coincides with the image of $\mathcal{L}$ in $\Gamma\left(\tilde{Y}, R^{1} f_{\tilde{Y}} \mathcal{O}_{X \times Y^{*}}^{*}\right)$. We call $\tau$ the universal $Y$-morphism defined by $\mathcal{L}$.

d) When $f$ admits a holomorphic section $s: Y \rightarrow X$ we have Pic $X / Y\left(Y^{\prime}\right)$ =the set of invertible sheaves $\mathcal{L}$ on $X \times_{Y} Y^{\prime}$ together with a fixed isomorphism $s^{*} \mathcal{L} \cong \mathcal{O}_{Y^{\prime}}$ where $s^{\prime}=s \times_{Y} i d_{Y^{\prime}}$ (cf. [13]). In this case the corresponding universal invertible sheaf $\mathcal{L}$ on $X \times_{Y}$ Pic $X / Y$ is called the relative Poincaré sheaf associated to $s$. When $Y$ is a point, giving an $s$ is equivalent to giving a fixed point $o \in X$. 
In this case we call $\mathcal{L}$ the (normalized) Poincaré sheaf associated to $o \in X$. In the general case let $p_{i}:$ Pic $X / Y \times_{Y} \mathrm{Pic} X / Y \rightarrow \operatorname{Pic} X / Y$ be the projections to the $i$-th factors. Let $\mathcal{L}_{i}=\left(i d_{X} \times p_{i}\right) * \mathcal{L}$ which are invertible sheaves on $\hat{X}:=X \times_{Y} \mathrm{Pic} X / Y \times_{Y} \mathrm{Pic} X / Y$. Then $a_{X / Y}$ is nothing but the universal $Y$-morphism defined by $\mathcal{L}_{1} \otimes_{\mathcal{O}} \mathcal{L}_{2}^{-1}, \mathcal{O}=\mathcal{O}_{\hat{X}}$.

1.2. Div $X / Y$ and Pic $X / Y$. a) Let $f: X \rightarrow Y$ be as in 1.1. Define a contravariant functor $\operatorname{Div} X / Y:(\operatorname{An} / Y) \rightarrow($ Sets $)$ by $\operatorname{Div} X / Y\left(Y^{\prime}\right)=$ the set of all effective relative divisors $Z \cong X \times_{Y} Y^{\prime}$ over $Y^{\prime}$ where a relative divisor is a Cartier divisor which is fiat over $Y^{\prime}$. Then $\operatorname{Div} X / Y$ is represented by a Zariski open subset $\operatorname{Div} X / Y$ of $D_{X / Y}$ which is a union of connected components where $D_{X / Y}$ is the relative Douady space of $X$ over $Y$ (cf. [5]). We write $\delta=\delta_{X / Y}$ : $\operatorname{Div} X / Y \rightarrow Y$ for the structural morphism.

b) Let $Z_{X / Y} \subseteq X \times_{Y} \operatorname{Div} X / Y$ be the universal relative divisor over $\operatorname{Div} X / Y$. We note that $\operatorname{Div} X / Y$ has the natural structure of a relative complex semigroup over $Y$ induced by the universality. We denote by $\tilde{m}_{X / Y}$ : $\operatorname{Div} X / Y{ }_{{ }_{Y}} \operatorname{Div} X / Y$ $\rightarrow \operatorname{Div} X / Y$ the corresponding multiplication. When $Y$ is a point, we write $\operatorname{Div} X$ for $\operatorname{Div} X / Y$.

c) Let $\left[Z_{X / Y}\right]$ be the line bundle on $X \times_{Y} \operatorname{Div} X / Y$ defined by $Z_{X / Y}$. Then we denote by $\mu_{X / Y}: \operatorname{Div} X / Y \rightarrow \operatorname{Pic} X / Y$ the universal $Y$-morphism defined by $\left[Z_{X / Y}\right]$. Suppose that $f$ admits a holomorphic section so that the relative Poincaré sheaf $\mathcal{L}$ exists. Then by Grothendieck ([13], exposé 232, Th. 4.3) there exists a coherent analytic sheaf $Q$ on Pic $X / Y$ such that $\operatorname{Div} X / Y$ is isomophic to the projective variety associated to $Q$ (cf. Fischer [4] p. 55). $Q$ is in fact given by $\left(V_{\mathrm{Pic} X / Y}\right)^{-1}\left(\mu_{X / Y}\right)_{+} L^{v}$ in the notation of Schuster [22]*) where $L^{2}$ denotes the line bundle dual to the line bundle corresponding to $\mathcal{L}$. In general, since $f$ admits a local section at any point of $Y$, this implies that $y \in Y$ has a neighborhood $V$ over which $\mu_{X / Y}$ is projective, and that the fiber over any $p \in \mathrm{Pic} X / Y$ is isomorphic to the projective space $\boldsymbol{P}\left(\Gamma\left(X_{b(p)}, L_{p}\right)\right):=\left(\Gamma\left(X_{b(p)}, L_{p}\right)\right.$ $-\{0\}) / C^{*}$. In particular if $\operatorname{dim} \Gamma\left(X_{b(p)}, L_{p}\right)=k+1$ is independent of $p \in N$ for some open subset $N \cong \operatorname{Pic} X / Y$, then $\mu_{X / Y}$ is a holomorphic $\boldsymbol{P}^{k}$-bundle when restricted over $N$.

1.3. Pic $X / Y$ in a special case. We now consider Pic $X / Y$ in the case where $f$ is a fiber space and $X_{y} \in \mathcal{C}$ for all $y \in Y$. In this case a direct construction of Pic $X / Y$ is known (cf. [14]), and is roughly described as follows.

a) The construction. We set $E_{1}=R^{1} f_{*} C / H^{1,0}$ and $E_{2}=R^{2} f_{*} C /\left(H^{2,0} \oplus H^{1,1}\right)$ where $H^{p, q}$ is the Hodge subbundles of type $(p, q)$. Then $E_{i}$ are holomorphic vector bundles over $Y$ such that $\mathcal{O}_{Y}\left(E_{i}\right) \cong R^{i} f_{*} \mathcal{O}_{X}$ naturally. Set $L_{i}=R^{i} f_{*} Z$, $i=1,2$. Then the inclusion $\boldsymbol{Z} \cong \boldsymbol{C}$ induces the natural homomorphisms $j_{i}: L_{i} \rightarrow E_{i}$

*) For any complex space $X, V_{X}: \operatorname{Coh}_{X} \rightarrow \operatorname{Lin}_{X}$ denotes the natural anti-equivalence where $\operatorname{Coh}_{X}$ (resp. $\operatorname{Lin}_{X}$ ) is the category of coherent analytic sheaves (resp. of linear fiber spaces) on $X$ (cf. [4], 1.6, [22], §3). 
where we consider $L_{i}, E_{i}$ as relative complex Lie groups over $Y$. Then it turns out that $j_{1}$ is injective. We set $\mathrm{Pic}_{0} X / Y:=E_{1} / L_{1}$. $\mathrm{Pic}_{0} X / Y$ is thus smooth over $Y$ with $\left(\operatorname{Pic}_{0} X / Y\right)_{y}=\mathrm{Pic}_{0} X_{y}$ for $y \in Y$. Moreover we obtain an exact sequence

$$
0 \longrightarrow \operatorname{Pic}_{0} X / Y \longrightarrow \operatorname{Pic} X / Y \stackrel{c_{1}}{\longrightarrow} L_{2} \stackrel{j_{2}}{\longrightarrow} E_{2}
$$

of relative complex Lie groups over $Y$ such that taking the sheaves of germs of holomorphic sections of these groups we obtain an exact sequence of $\mathcal{O}_{Y^{-}}$ modules

$$
0 \longrightarrow R^{1} f_{*} \mathcal{O}_{X} / R^{1} f_{*} Z \longrightarrow R^{1} f_{*} \mathcal{O}_{X}^{*} \longrightarrow R^{2} f_{*} Z \longrightarrow R^{2} f_{*} \mathcal{O}_{X}
$$

coming from the usual exponential sequence $0 \rightarrow \boldsymbol{Z} \rightarrow \mathcal{O}_{X} \rightarrow \mathcal{O}_{X}^{*} \rightarrow 0$ where for $p \in \operatorname{Pic} X / Y, c_{1}(p)=c_{1}\left(L_{p}\right) \in H^{2}\left(X_{b(p)}, Z\right)=\left(R^{2} f_{*} Z\right)_{b(p)}$.

b) Essential component. Let $L_{\gamma}, \gamma \in \Gamma^{\prime}(f)$, be the set of connected componets of $L_{2}$. Here a special index $0 \in \Gamma^{\prime}(f)$ is specified by the condition that $L_{0}$ is the zero section of $\varepsilon: L_{2} \rightarrow Y$. Let $L_{\gamma}(\underline{0}):=j_{2}^{-1}(\underline{0}) \cap L_{\gamma}$, where $\underline{0}$ denotes the zero section of $E_{2}$. Let $\operatorname{Pic}_{\gamma} X / Y:=c_{1}^{-1}\left(L_{\gamma}(\underline{0})\right.$ ) (in compatible with the above definition of $\left.\operatorname{Pic}_{0} X / Y\right)$. Then $c_{1}$ induces a proper smooth morphism $c_{1}(\gamma): \operatorname{Pic}_{\gamma} X / Y \rightarrow L_{\gamma}(\underline{0})$, the fiber over $q \in L_{\gamma}(\underline{0})$ being isomorphic to a connected component $\operatorname{Pic}_{\gamma(q)} X_{y}$ of $\operatorname{Pic} X_{y}$ consisting of those line bundles whose chern class is $q \in L_{\gamma, y} \cong H^{2}\left(X_{y}, \boldsymbol{Z}\right)$ where $y=\varepsilon(q)$. In particular if $j_{2}\left(L_{\gamma}\right)=\underline{0}$, i. e., $L_{\gamma}=L_{\gamma}(\underline{0})$, then $\mathrm{Pic}_{\gamma} X / Y$ is connected and the natural map $b_{\gamma}: \operatorname{Pic}_{\gamma} X / Y \rightarrow Y$ is smooth since $L_{\gamma} \rightarrow Y$ is unramified. We call such a component $\mathrm{Pic}_{\gamma} X / Y$ an essential component of $\mathrm{Pic} X / Y$. An essential component is precisely a component which is mapped surjectively onto $Y$.

We denote by $\left\{\mathrm{Pic}_{\gamma} X / Y\right\}, \gamma \in \Gamma(f)$, the set of essential components of Pic $X / Y$. When $Y$ is a point we write $\operatorname{Pic}_{\gamma} X$ instead of $\operatorname{Pic}_{\gamma} X / Y$. In this case we have the natural identification of $\Gamma(f)$ with the Neron-Severi group $N S(X)$ of $X$, the group of the first chern classes of line bundles on $X$.

c) Some remarks. i) Let $Y^{\prime} \cong Y$ be any Zariski open subset. Then the restriction $\mathrm{Pic}_{\gamma} X / Y \rightarrow \mathrm{Pic}_{\gamma} X_{Y^{\prime}} / Y^{\prime}$ sets up a natural bijective correspondence between the sets of essential components of Pic $X / Y$ and Pic $X_{Y^{\prime}} / Y^{\prime}$ where $X_{Y^{\prime}}$ $=X \times_{Y} Y^{\prime}$. In particular we can naturally identify $\Gamma\left(f_{Y^{\prime}}\right)$ with $\Gamma(f)$.

ii) If for some $\gamma \in \Gamma(f), L_{\gamma} \rightarrow Y$ is finitely unramified of degree $m$ we can associate canonically to $L_{\gamma}$ another connected component $L_{a(\gamma)}$ for which $L_{a(\gamma)} \rightarrow Y$ is isomorphic; if $L_{\gamma, y}=\left\{t_{1}(y), \cdots, t_{m}(y)\right\}$, then $y \rightarrow \sum_{i=1}^{m} t_{i}(y)$ defines a holomorphic section $s: Y \rightarrow L_{2}$ and we simply set $L_{a(\gamma)}=s(Y)$. In this case the corresponding $\operatorname{Pic}_{a(\gamma)} X / Y \rightarrow Y$ is a smooth fiber space.

iii) Let $\nu: \tilde{Y} \rightarrow Y$ be a surjective morphism of complex varieties. Then we have a unique map $\Gamma(\nu): \Gamma(\tilde{f}) \rightarrow \Gamma(f)$ determined by the condition that $P_{\gamma}(\nu)$ : $\operatorname{Pic}_{\gamma} X / Y \times_{Y} \tilde{Y} \cong \coprod_{\tilde{Y}} \operatorname{Pic}_{\tilde{\gamma}} \tilde{X} / \tilde{Y}, \tilde{\gamma} \in \Gamma(\nu)^{-1}(\gamma)$, with respect to the isomorphism $P$ in 1.1 b) where $\tilde{X}=X \times_{Y} \tilde{Y}$ and $\tilde{f}: \tilde{X} \rightarrow \tilde{Y}$ is the natural morphism. 
iv) Let $f^{\prime}: X^{\prime} \rightarrow Y$ and $g: X^{\prime} \rightarrow X$ be as in $\left.1.1 \mathrm{~b}\right)$ ii). Then we have the natural morphism $\Gamma(g): \Gamma(f) \rightarrow \Gamma\left(f^{\prime}\right)$ by the conidition that $g^{*}\left(\mathrm{Pic}_{\gamma} X / Y\right)$ $\subseteq \mathrm{Pic}_{\Gamma(g) r} X^{\prime} / Y$. Of course we have $\Gamma(g)(0)=0$.

v) For $\gamma \in \Gamma(f)$ we set $\operatorname{Div}_{\gamma} X / Y=\left(\mu_{X / Y}^{-1}\left(\operatorname{Pic}_{\gamma} X / Y\right)\right)_{r e d}$ and $\mu_{\gamma}:=\left.\mu_{X / Y}\right|_{\operatorname{Div}_{\gamma} X / Y}$ : $\operatorname{Div}_{\gamma} X / Y \rightarrow \operatorname{Pic}_{\gamma} X / Y$. We denote by $Z_{Y}=\left(Z_{X / Y}\right)_{\gamma}$ the universal divisor restricted to $\operatorname{Div}_{\gamma} X / Y . \quad m_{X / Y}, a_{X / Y}$ defined in 1.1 a) defines $Y$-morphsims $m_{\gamma, r^{\prime}}$ : $\operatorname{Pic}_{\gamma} X / Y \times_{Y} \mathrm{Pic}_{\gamma^{\prime}} X / Y \rightarrow \mathrm{Pic}_{\gamma+\gamma^{\prime}} X / Y \quad$ (resp. $a_{\gamma, \gamma^{\prime}}: \mathrm{Pic}_{\gamma} X / Y \times_{Y} \mathrm{Pic}_{\gamma^{\prime}} X / Y \rightarrow \mathrm{Pic}_{\gamma-\gamma^{\prime}} X / Y$ ) where $\gamma, \gamma^{\prime} \in \Gamma(f)$; in this way $\Gamma(f)$ itself has the natural structure of an additive group with the identity $0 \in \Gamma(f)$.

\subsection{Relative Albanese map (smooth case).}

Definition 1. Let $f: X \rightarrow Y$ be a smooth fiber space of complex varieties. Then a relative Albanese map for $f$ is a commutative diagram of complex varieties

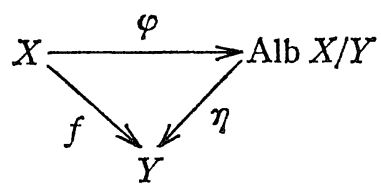

where $\eta$ is a smooth fiber space with any fiber a complex torus and $\varphi$ is a $Y$ morphism, with the following universal property: Let $Y^{\prime}$ be any complex variety over $Y, T^{\prime} \rightarrow Y^{\prime}$ any smooth morphism with any fiber a complex torus and $\varphi^{\prime}: X \times_{Y} Y^{\prime} \rightarrow T^{\prime}$ any $Y^{\prime}$-morphism. Then there exists a unique $Y^{\prime}$-morphism $h^{\prime}:$ Alb $X / Y \times_{Y} Y^{\prime} \rightarrow T^{\prime}$ such that $\varphi^{\prime}=h^{\prime} \varphi_{Y^{\prime}}$. We often call $\varphi$ itself the relative Albanese map for $f$ and $\operatorname{Alb} X / Y$ the relative Albanese variety for $f$.

From the definition the following is true: (P) For any $y \in Y, \varphi_{y}: X_{y}$ $\rightarrow(\operatorname{Alb} X / Y)_{y}$ is isomorphic to the Albanese map $\varphi(y): X_{y} \rightarrow \operatorname{Alb} X_{y}$ of $X_{y}$. On the existence of the relative Albanese variety we have the following:

Proposition 1. Let $f: X \rightarrow Y$ be a smooth fiber space of complex varieties such that $X_{y} \in \mathcal{C}$ for some $y \in Y$. Then a relative Albanese map for $f$ exists. Moreover it is up to isomorphisms uniquely characterized by the property $(P)$ above.

See [12] for the proof. In fact, we need the proposition only in the case where $X_{y} \in \mathcal{C}$ for all $y \in Y$ and in this case the construction is easy, though we need not the construction itself here (cf. [13] 236 and the proof of Theorem 1 below).

1.5. s. ampleness. Let $X$ be a compact complex manifold. We call $\gamma \in N S(X)$, or any line bundle $L \in \mathrm{Pic}_{\gamma} X$, s. ample (sufficiently ample) if $L^{\prime}$ is very ample and $H^{i}\left(X, L^{\prime}\right)=0, i>0$, for any $L^{\prime} \in \mathrm{Pic}_{\gamma} X$. For any ample line bundle $L_{1}$ its high multiple is always s. ample (cf. Kodaira [17]). Note that the definition of 
s. ampleness naturally extends to any compact complex space (not necessarily reduced).

Let $f: X \rightarrow Y$ be a smooth fiber space of compact complex varieties in $\mathcal{C}$. Then an essential component $\operatorname{Pic}_{\gamma} X / Y$, or the index $\gamma \in \Gamma(f)$ itself, is called s. ample if there exists a Zariski open subset $U_{\gamma} \subseteq Y$ such that for any $p \in b_{\gamma}^{-1}\left(U_{\gamma}\right)$ the corresponding line bundle $L_{p}$ on $X_{b(p)}$ is s. ample. In general, if $L_{p}$ is $\mathrm{s}$. ample for all $p \in N$ where $N$ is an open subset of $\operatorname{Pic} X / Y$, then $\operatorname{dim} \Gamma\left(X_{b(p)}, L_{p}\right)$ $=k+1$ is independent of $p \in N$. Hence by 1.2 c) $\mu_{\gamma}: \operatorname{Div}_{\gamma} X / Y \rightarrow \operatorname{Pic}_{\gamma} X / Y$ is a holomorphic $\boldsymbol{P}^{k}$-bundle over $N$. In particular this is the case with $N=b_{\gamma}^{-1}(U)$ if $\gamma$ is s. ample.

1.6. Description of Albanese map. a) Let $X$ be a projective manifold. Fix a base point $o \in X$. Let $\mathcal{L} \rightarrow X \times$ Pic $X$ be the Poincaré sheaf associated to $X$ and $o \in X$. Then $\mathcal{L}_{0} \rightarrow X \times \operatorname{Pic}_{0} X$, considered as a family of invertible sheaves on $\mathrm{Pic}_{0} X$ parametrized by $X$, defines the universal morphism $\psi: X \rightarrow \mathrm{Pic}_{0} \mathrm{Pic}_{0} X$. Then $\psi$ is naturally identified with an Albanese map $\phi_{X}: X \rightarrow \operatorname{Alb} X$ of $X$ (cf. [13] exposé 236). Let $f: X \rightarrow X^{\prime}$ be a morphism of projective manifolds. Since $\mathrm{Pic}_{0}$ is contravariant, we get a homomorphism $F: \mathrm{Pic}_{0} \mathrm{Pic}_{0} X \rightarrow \mathrm{Pic}_{0} \mathrm{Pic}_{0} X^{\prime}$. Then we have $F \phi_{X}=\phi_{X^{\prime}} f$.

b) Fix an s. ample $\gamma \in N S(X)$ so that $\operatorname{Div}_{\gamma} X$ is a holomorphic $\mathbb{P}^{k}$-bundle over $\operatorname{Pic}_{\gamma} X$ for some $k>0$. Let $Z_{\gamma} \subseteq X \times \operatorname{Div}_{\gamma} X$ be the universal divisor. To obtain another description of Albanese map first we prove the following:

Lemma 1. Consider $Z_{\gamma, o} \subseteq\{0\} \times \operatorname{Div}_{\gamma} X \subseteq \operatorname{Div}_{\gamma} X$ as a divisor on $\operatorname{Div}_{\gamma} X$ and set $\tilde{Z}_{\gamma, 0}=X \times Z_{\gamma, o} \subseteq X \times \operatorname{Div}_{\gamma} X$. Let $\mathscr{T}_{\gamma}=\mathcal{O}\left(\left[Z_{\gamma}\right]\right)$ and $\mathscr{T}_{\gamma}^{\prime}=\mathcal{O}\left(\left[\tilde{Z}_{\gamma, o}\right]\right)$ where $\mathcal{O}=\mathcal{O}_{X \times \operatorname{Div}_{\gamma} X}$. Then $\mathscr{I}_{\gamma} \otimes_{\mathcal{O}} \mathscr{I}_{\gamma}^{\prime 2} \cong\left(i d_{X} \times \mu_{\gamma}\right)^{*} \mathcal{L}_{\gamma}$, so that in particular $\left(i d_{X} \times \mu_{\gamma}\right)_{*}\left(\mathscr{I}_{\gamma} \otimes_{\mathcal{O}} \mathscr{T}_{\gamma}^{\prime 2}\right) \cong \mathcal{L}_{\gamma}$ where $\mathscr{F}_{\gamma}^{\prime}=\operatorname{Hom}_{\mathcal{O}}\left(\mathscr{I}_{\gamma}^{\prime}, \mathcal{O}\right)$.

Proof. Let $\mathcal{E}_{\gamma}=\mathscr{F}_{\gamma} \otimes_{\odot} \mathscr{I}_{\gamma}^{\prime 2}$. Since $\mathcal{E}_{\gamma}$ is trivial when restricted to each fiber of $i d_{X} \times \mu_{\gamma}$, and $i d_{X} \times \mu_{\gamma}$ is a $\boldsymbol{P}^{k}$-bundle, there exists a unique invertible sheaf $\mathscr{M}_{\gamma}$ on $X \times \operatorname{Pic}_{\gamma} X$ such that $\mathcal{E}_{\gamma}=\left(i d_{X} \times \mu_{\gamma}\right) * \mathscr{M}_{\gamma}$. It suffices to show that $\mathcal{L}_{\gamma} \cong \mathscr{M}_{\gamma}$. By the definitions of these sheaves and of $Z_{\gamma}$ we infer readily that 1) for any $p \in \operatorname{Pic}_{\gamma} X, \mathscr{M}_{\gamma, p} \cong \mathcal{O}_{X}\left(\left[Z_{\gamma, d}\right]\right) \cong \mathcal{L}_{\gamma, p}$ on $X=X \times\{p\}=X \times\{d\}$ where $d \in\left(\operatorname{Div}_{\gamma} X\right)_{p}$ is an arbitrary point, and 2) $\mathcal{L}_{\gamma, 0} \cong \mathcal{O}_{\mathrm{Pic}_{\gamma} X} \cong \mathscr{M}_{\gamma, 0}$ on $\mathrm{Pic}_{\gamma} X=\{0\} \times \mathrm{Pic}_{\gamma} X$. From this it follows immediately that $\mathscr{M}_{7} \cong \mathcal{L}_{7}$.

q. e. d.

c) In the notation of b) $Z_{\gamma}$ is a relative divisor also over $X$ since $\gamma$ is s. ample. Let $\varphi: X \rightarrow \operatorname{Div}\left(\operatorname{Div}_{\gamma} X\right)$ be the associated universal morphism which factors through $\operatorname{Div}_{\tilde{o}}\left(\operatorname{Div}_{\gamma} X\right) \subseteq \operatorname{Div}\left(\operatorname{Div}_{\gamma} X\right)$ for a unique $\delta \in N S\left(\operatorname{Div}_{\gamma} X\right)$. The resulting morphism $X \rightarrow \operatorname{Div}_{\tilde{o}} \operatorname{Div}_{\gamma} X$ will still be denoted by $\varphi$.

Lemma 2. Let $\psi^{\prime}:=\mu_{\hat{\delta}} \varphi: X \rightarrow \operatorname{Pic}_{\tilde{o}}\left(\operatorname{Div}_{\gamma} X\right)$ where $\mu_{\delta}: \operatorname{Div}_{\tilde{o}}\left(\operatorname{Div}_{\gamma} X\right) \rightarrow \operatorname{Pic}_{\tilde{\delta}}\left(\operatorname{Div}_{\gamma} X\right)$ is the natural morphism. Then $\psi^{\prime}$ is an Albanese map of $X$.

Proof. Let $\psi_{\gamma}: X \rightarrow \mathrm{Pic}_{0}\left(\mathrm{Pic}_{\gamma} X\right)$ be the morphism defined by the universality 
of the Poincaré sheaf $\mathcal{L}_{\gamma} \rightarrow X \times \mathrm{Pic}_{\gamma} X$, i. e., $\phi_{\gamma}(x)$ is the point corresponding to the invertible sheaf $\mathcal{L}_{\gamma, x}$ on $\mathrm{Pic}_{\gamma} X$ (which has the zero chern class). Let $j: \operatorname{Pic}_{\hat{o}}\left(\operatorname{Div}_{\gamma} X\right) \rightarrow \operatorname{Pic}_{0}\left(\operatorname{Div}_{r} X\right)$ be the isomorphism defined by the subtraction by $\phi^{\prime}(o)$. Let $\eta_{\gamma}: \operatorname{Pic}_{0}\left(\operatorname{Pic}_{\gamma} X\right) \rightarrow \operatorname{Pic}_{0}\left(\left(\operatorname{Div}_{\gamma} X\right)\right.$ be the isomorphism induced by $\mu_{\gamma}$. Then we show that $\phi^{\prime \prime}:=\eta{ }_{r}^{-1} j \psi^{\prime}: X \rightarrow \mathrm{Pic}_{0} \mathrm{Pic}_{\gamma} X$ coincides with $\phi_{r}$ above. First, let $F_{x}$ be the line bundle on $\mathrm{Pic}_{\gamma} X$ corresponding to $\psi^{\prime}(x) \in \operatorname{Pic}_{\tilde{\delta}}\left(\operatorname{Pic}_{\gamma} X\right)$ and $Z_{x}$ the divisor on $\operatorname{Div}_{\gamma} X$ corresponding to $\varphi(x) \in \operatorname{Div}_{\delta}\left(\operatorname{Div}_{\gamma} X\right)$ so that $F_{x} \cong\left[Z_{x}\right]$. Then from the definition of $\psi^{\prime \prime}$ it follows that $\psi^{\prime \prime}(x)$ is the unique line bundle $M_{x}$ on $\mathrm{Pic}_{\gamma} X$ satisfying $\mathscr{F}_{x} \otimes \mathscr{F}_{0}^{-1} \cong \mu_{\gamma}^{*} \mathscr{M}_{x}$ where $\mathscr{F}_{x}=\mathcal{O}_{\operatorname{Div}_{\gamma} X}\left(F_{x}\right)$ and $\mathscr{M}_{x}=\mathcal{O}_{\mathrm{Pic}_{\gamma} X}\left(M_{x}\right)$. Then it suffices to show that $\mathscr{M}_{x} \cong \mathcal{L}_{\gamma}$, which is in fact the case by virtue of Lemma 1. Since $\psi_{r}$ is naturally isomorphic to $\phi_{X}: X \rightarrow \mathrm{Pic}_{0} \mathrm{Pic}_{0} X, \psi_{r}$ is an Albanese map of $X$ by a).

q. e. d.

\section{§ 2. The Structure of $\operatorname{Div}^{*} X / Y$ and Relative Algebraic Reduction}

2.1. $\operatorname{Div}^{*} X / Y$. Let $f: X \rightarrow Y$ be a generically smooth fiber space of compact complex varieties in $\mathcal{C}$. Let $U \subseteq Y$ be a Zariski open subset over which $f$ is smooth. We write $X_{U}:=f^{-1}(U)$ and $f_{U}:=\left.f\right|_{f^{-1}(U)}: X_{U} \rightarrow U$.

a) We shall fix some notations which will be used also in Section 3.

i) Let $\mathrm{Pic}_{\gamma} X_{U} / U, \gamma \in \Gamma\left(f_{U}\right)$, be the essential components of Pic $X_{U} / U$ (cf. 1.3). In view of $1.3 \mathrm{c}$ ) i) if $U^{\prime}$ is another Zariski open subset over which $f$ is smooth, we can naturally identify the index sets $\Gamma\left(f_{U}\right)$ and $\Gamma\left(f_{U^{\prime}}\right)$. So in what follows we may, and we shall, denote $\Gamma\left(f_{U}\right)$ for any $U$ as above by $\Gamma(f)$.

ii) Let $\nu: \tilde{Y} \rightarrow Y$ be a surjective morphism with $\tilde{Y}$ a compact complex variety in $\mathcal{C}$. We set $\tilde{X}=X \times_{Y} \tilde{Y}, \tilde{U}=\nu^{-1}(U)$ and $\tilde{f}=f \times_{Y} \tilde{Y}: \tilde{X} \rightarrow \tilde{Y}$. Then we obtain a natural map $\Gamma(\nu): \Gamma(\tilde{f}) \rightarrow \Gamma(f)$ by 1.3 c) iii), in view of the above definition of $\Gamma(f)$ and $\Gamma(\tilde{f})$.

iii) Let $f^{\prime}: X^{\prime} \rightarrow Y$ be another fiber space of compact complex varieties in $\mathcal{C}$ which is smooth over $U$. Let $g: X^{\prime} \rightarrow X$ be a meromorphic $Y$-map which is holomorphic over $U$. Then in view of $1.3 \mathrm{c}$ ) iv) and the definition of $\Gamma(f)$ and $\Gamma\left(f^{\prime}\right), g$ induces a unique map $\Gamma(g): \Gamma(f) \rightarrow \Gamma\left(f^{\prime}\right)$.

b) The Zariski open subset $\operatorname{Div}_{\gamma}\left(X_{U} / U\right) \subseteq D_{X_{U} / U, \text { red }}$ is also Zariski open in $D_{X / Y, \text { red }}$ (cf. 1.2 a)). Let $\operatorname{Div}_{\gamma}^{-} X / Y$ be the closure of $\operatorname{Div}_{\gamma} X_{U} / U$ in $D_{X / Y \text {, red }}$ and $\operatorname{Div}_{\gamma}^{*} X / Y$ the union of those irreducible components of $\operatorname{Div}_{\gamma}^{-} X / Y$ which are mapped surjectively onto $Y$. Then it is readily seen that $\operatorname{Div}_{\gamma}^{*} X / Y$ is independent of the choice of $U$ as above (as a subspace of $D_{X / Y}$ ). Let $Z_{\gamma}^{*}$ be the closure of $Z_{\gamma} \subseteq X_{U} \times_{U} \operatorname{Div}_{\gamma} X_{U} / U$ in $X \times_{Y} \operatorname{Div}_{\gamma}^{*} X / Y$ which is again analytic and proper over $Y$. We call $Z_{\gamma}^{*}$ the meromorphic universal relative divisor for each $\gamma$. $Z_{\gamma}^{*}$ neither depends on the choice of $U$ as above. We further set $\operatorname{Div}^{*} X / Y$ $=\bigcup_{\gamma \in \Gamma(f)} \operatorname{Div}_{\gamma}^{*} X / Y$. We also recall that $\operatorname{Div}_{\gamma}^{*} X / Y$ is a compact complex space in $\mathcal{c}$ (cf. [6]).

c) As follows easily from the definition the formation of $\operatorname{Div}^{*} X / Y$ has the 
following properties.

i) If $\nu: \tilde{Y} \rightarrow Y$ is as in a) ii), then we have the natural isomorphism $D_{\gamma}^{*}(\nu)$ : $\operatorname{Div}_{\gamma}^{*} X / Y \dot{\times}_{Y} \tilde{Y} \cong \cup_{\tilde{\gamma}} \operatorname{Div}_{\tilde{\gamma}}^{*} \tilde{X} / \tilde{Y}, \tilde{\gamma} \in \Gamma(\nu)^{-1}(\gamma)$, and hence $\operatorname{Div}^{*} X / Y \dot{\times}_{Y} \tilde{Y} \cong \operatorname{Div}^{*} \tilde{X} / \tilde{Y}$ with respect to the natural isomorphism $D_{X / Y} \times_{Y} \tilde{Y} \cong D_{\tilde{X} / \tilde{Y}}$ where $\dot{X}$ denotes the strict pull-back (cf. Convention).

ii) Let $f^{\prime}: X^{\prime} \rightarrow Y$ and $g: X^{\prime} \rightarrow X$ be as in a) iii) above. Let $\delta_{\gamma}^{*}: \operatorname{Div}_{\gamma}^{*} X / Y \rightarrow Y$ be the structure morphism. If for general $d \in \operatorname{Div}_{\gamma}^{*} X / Y, g\left(X^{\prime}\right)_{y} \nsubseteq Z_{\gamma, d}^{*}\left(y=\delta_{\gamma}^{*}(d)\right)$, then $g$ induces a natural meromorphic $Y$-map $\tilde{g}_{\gamma}^{*}: \operatorname{Div}_{\gamma}^{*} X / Y \rightarrow \operatorname{Div}_{\gamma^{\prime}}^{*} X^{\prime} / Y$ with $\gamma^{\prime}=\Gamma(g) \gamma$ and hence a meromorphic $Y$-map $\tilde{g}^{*}: \operatorname{Div}^{*} X / Y \rightarrow \operatorname{Div}^{*} X^{\prime} / Y$.

iii) There exists a meromorphic $Y$-map $\tilde{m}_{\gamma, \gamma^{\prime}}^{*}: \operatorname{Div}_{\gamma}^{*} X / Y \times_{Y} \operatorname{Div}_{\gamma^{\prime}}^{*} X / Y$ $\rightarrow \operatorname{Div}_{\gamma+\gamma^{\prime}}^{*} X / Y, \gamma, \gamma^{\prime} \in \Gamma(f)$, which is bimeromorphic over $U$ to the $U$-morphism $\tilde{m}_{\gamma, \gamma^{\prime}}: \operatorname{Div}_{\gamma} X_{U} / U \times_{U} \operatorname{Div}_{\gamma^{\prime}} X_{U} / U \rightarrow \operatorname{Div}_{\gamma+\gamma^{\prime}} X_{U} / U$ induced by $\tilde{m}_{X / Y}$ (cf. $\left.\left.1.2 \mathrm{~b}\right)\right)$.

\subsection{Some lemmas on s. ample components.}

Lemma 3. Let $f: X \rightarrow T$ be a proper morphism of compact complex varieties. Let $\mathscr{I}$ be a coherent analytic sheaf on $X$. Suppose that there exists a Zariski open subset $U \subseteq T$ such that $\subseteq$ is invertible on $X_{U}$ and $f$ is flat on $X_{U}$. Suppose further that there exists $o \in U$ such that $\mathscr{F}_{o}$ is s. amples on $X_{0}$. Then there exists a Zariski open subset $V \leqq T$ such that $V \leqq U$ and $V=\left\{t \in U ; \mathscr{I}_{t}\right.$ is s. ample on $\left.X_{t}\right\}$.

Proof. If $f$ is flat and $\mathscr{I}$ is invertible on the whole $X$, then by the Zariski openness of very ampleness and the upper semicontinuity of cohomology dimension on the fibers, it is immediate to see that the set $T^{\prime}:=\left\{t \in T ; \mathscr{F}_{t}\right.$ is $\mathrm{s}$. ample $\}$ itself is Zariski open. Then we have only to set $V=T^{\prime} \cap U$. In the general case take a proper modification $\sigma_{1}: X_{1} \rightarrow X$ such that the strict transform $\mathscr{I}_{1}$ of I on $X_{1}$ is invertible and that $\sigma_{1}$ gives an isomorphism of $\sigma_{1}^{-1}\left(X_{U}\right)$ and $X_{U}$ [21]. Let $\eta: T_{2} \rightarrow T$ be a proper modification such that $\left.\eta\right|_{\eta^{-1}(U)}: \eta^{-1}(U) \rightarrow U$ is isomorphic and that the strict transform $X_{2}$ of $X_{1}$ in $X_{1} \times_{T} T_{2}$ is flat over $T_{2}$ ([15]). Let $\mathscr{I}_{2}$ be the pull-back of $\mathscr{T}_{1}$ to $X_{2}$. Then $T_{2}^{\prime}:=\left\{t \in T_{2} ; \mathscr{T}_{2, t}\right.$ is s. ample $\}$ is Zariski open in $T_{2}$ as above. Then we have only to set $V=\eta\left(T_{2}^{\prime} \cap \eta^{-1}(U)\right)$. q. e.d.

Lemma 4. Let $f: X \rightarrow Y$ be a generically smooth fiber space of compact complex varieties in $\mathcal{C}$. Let $U \subseteq Y$ be a Zariski open subset over which $f$ is smooth. Let $\mathrm{Pic}_{\gamma} X_{U} / U$ be an essential component of $\mathrm{Pic} X_{U} / U$. If there exists a point $p \in \mathrm{Pic}_{\gamma} X_{U} / U$ such that the corresponding line bundle $L_{p}$ on $X_{b(p)}$ is s. ample, then $\mathrm{Pic}_{\gamma} X_{U} / U \rightarrow U$ is proper, smooth and $\gamma$ is s. ample.

Proof. Let $y=b(p)$. Write for simplicity $P_{\gamma}=\mathrm{Pic}_{\gamma} X_{U} / U$. Let $P_{\gamma, y, 1}$ be a connected component of $P_{r, y}$ with $p \in P_{r, y, 1}$ so that every point of $P_{r, y, 1}$ corresponds to an s. ample line bundle on $X_{b(p)}$. Then there exists a Zariski open neighborhood $N$ of $P_{\gamma, y, 1}$ in $P_{\gamma}$ such that for any $q \in N$, the corresponding line bundle $L_{q}$ on $X_{b(q)}$ is s. ample (cf. the proof of the previous lemma). Then since $\mu_{\gamma}\left(\operatorname{Div}_{\gamma} X_{U} / U\right) \supseteqq N$ and $\delta_{X_{U} / U}=b_{X_{U} / U} \mu_{X_{U} / U}, \quad \delta_{\gamma}^{*}\left(\operatorname{Div}_{\gamma}^{*} X / Y\right)$ contains an open 
subset of $Y$. Hence, being an analytic subset of $Y$, it coincides with $Y$. Write $D_{\gamma}^{*}=\operatorname{Div}_{\gamma}^{*} X / Y$. By the previous lemma applied to $X \dot{\times}_{Y} D_{\gamma}^{*} \rightarrow D_{\gamma}^{*}$ there exists a Zariski open subset $V \cong D_{\gamma}^{*}$ such that $V=\left\{d \in \operatorname{Div}_{\gamma} X_{U} / U ;\left[Z_{X / Y}\right]_{d}\right.$ is s. ample on $\left.X_{\delta(d)}\right\}$. On the other hand, by the definition of s. ampleness for any $u \in U$ and any connected component $D_{\gamma, u, k}^{*}$ of $D_{\gamma, u}^{*}$ we have either $D_{\gamma, y, k}^{*} \cap V=\varnothing$ or $D_{\gamma, y, k}^{*} \leqq V$. Then it is easy to find a Zariski open subset $U_{\gamma} \leqq Y$ contained in $U$ such that $L_{p}$ is $\mathrm{s}$. ample for any $p \in b_{\gamma}^{-1}\left(U_{\gamma}\right)$. Thus $\gamma$ is s. ample. Finally since $D_{r, U}^{*} \rightarrow P_{\gamma}$ is surjective and $D_{\gamma, U}^{*}$ is proper over $U, P_{\gamma}$ is proper and smooth over $U$ (cf. 1.3 b)).

q.e. d.

Let $f: X \rightarrow Y$ be a fiber space of complex varieties. We say that $f$ is locally projective if for any $y \in Y$ there exists a neighborhood $y \in V$ such that $f_{V}: X_{V} \rightarrow V$ is projective. We say that $f$ is generically locally projective if there exists a Zariski open subset $U \subseteq Y$ such that $f_{U}: X_{U} \rightarrow U$ is locally projective.

Lemma 5. Let $f: X \rightarrow Y$ be a generically smooth fiber space of compact complex varieties in $\mathcal{C}$. Let $U$ be a Zariski open subset over which $f$ is smooth. Suppose that $f$ is projective and smooth over an open subset $W \leqq U$. Then there exists an s. ample component $\mathrm{Pic}_{\gamma} X_{U} / U$ of $\mathrm{Pic} X_{U} / U$ such that $\mathrm{Pic}_{\gamma} X_{U} / U \rightarrow U$ is a smooth fiber space. In particular $f$ is generically locally projective.

Proof. Fix $y \in W$. Let $L$ be a line bundle of $X_{W}$ which is very ample with respect to $f_{W}: X_{W} \rightarrow W$ (restricting $W$ if necessary). Replacing $L$ by its high multiple we may assume that $\left.L\right|_{x_{y}}$ is s. ample. Let $s: W \rightarrow \operatorname{Pic} X_{W} / W$ be the holomorphic section defined by $L$. Let $\operatorname{Pic}_{\gamma} X_{U} / U, \gamma \in \Gamma\left(f_{U}\right)$, be the unique connected component of Pic $X_{U} / U \supseteqq \operatorname{Pic} X_{W} / W$ containing $s(W)$. Then by Lemma 4 $\gamma$ is s. ample. If $\mathrm{Pic}_{\gamma} X_{U} / U \rightarrow U$ is not a fiber space, we have only to replace $\mathrm{Pic}_{\gamma} X_{U} / U$ by $\mathrm{Pic}_{a(\gamma)} X_{U} / U$ (cf. 1.3 c)). In fact, we easily check that each point $p \in b_{a(\gamma)}^{-1}\left(U_{\gamma}\right)$ corresponds to an s. ample line bundle on $X_{b(p)}$ with $U_{\gamma}$ as in 1.5. It follows that $f_{U_{\gamma}}: X_{U_{\gamma}} \rightarrow U_{\gamma}$ is locally projective.

q. e. d.

Lemma 6. Let $f: X \rightarrow Y$ be a generically smooth fiber space of compact complex varieties in $\mathcal{C}$. Let $U \subseteq Y$ be a Zariski open subset over which $f$ is smooth. Suppose that $f$ is generically locally projective. Then for any $\gamma \in \Gamma(f)$ we can find s. ample elements $\alpha, \beta \in \Gamma(f)$ such that $\gamma=\alpha-\beta$ and that $a_{\alpha \beta}: \operatorname{Pic}_{\alpha} X_{U} / U$ $\times_{U} \mathrm{Pic}_{\beta} X_{U} / U \rightarrow \mathrm{Pic}_{\gamma} X_{U} / U$ is a fiber space.

Proof. Take and fix an s. ample $\alpha^{\prime}$ according to Lemma 5 so that in particular $\operatorname{Pic}_{\alpha^{\prime}} X_{U} / U$ is a fiber space over $U$. Fix any $p \in \mathrm{Pic}_{\alpha^{\prime}} X_{U} / U$ such that the corresponding line bundle $L_{p}$ is s. ample on $X_{b(p)}$. Let $y=b(p)$. Take any $q \in\left(\operatorname{Pic}_{\gamma} X_{U} / U\right)_{y}$. Then $L_{p}^{\otimes n} \otimes L_{q}$ is s. ample on $X_{y}$ for a sufficiently large $n$. Then we set $\alpha=n \alpha^{\prime}$ and $\beta=\alpha+\gamma$. Then $\alpha$ and $\beta$ are s. ample. This is clear for $\alpha$ and is true for $\beta$ by Lemma 4 since $L_{p}^{\otimes n} \otimes L_{q}=L_{r}$ for some $r \in\left(\operatorname{Pic}_{\beta} X_{U} / U\right)_{y}$. Finally, since $\operatorname{Pic}_{\alpha} X_{U} / U$ is a fiber space over $U$ as well as $\mathrm{Pic}_{\alpha^{\prime}} X_{U} / U$, it follows readily that $a_{\alpha \beta}$ also is a fiber space.

q. e. d. 
2.3. Local projectivity of $\operatorname{Div}_{\gamma}^{*} X / Y$.

Lemma 7. Let $f: X \rightarrow Y$ be a generically smooth fiber space of complex varieties. Let $V \cong Y$ be an open subset such that $X_{y}$ are smooth and projective for all $y \in V$. Then $f$ is projective over some open subset of $V$.

Proof. By assumption for any $y \in V$ there exist an irreducible component $D(y)$ of $\operatorname{Div}^{-} X / Y$ (where $\operatorname{Div}^{-} X / Y$ is the closure of $\operatorname{Div}_{U} X_{U} / U$ in $D_{X / Y}$ with $U$ as in 2.1) and a point $d=d(y) \in D(y)_{y}$ such that the corresponding divisor $Z_{d}$ on $X_{y}$ is ample. Then we have $V \cong \bigcup_{y \in V} \delta(D(y))$. Since $\operatorname{Div}^{-} X / Y$ is countable (cf. 1.3), by Baire argument $V \cong \delta\left(D\left(y_{0}\right)\right)$ for some $y_{0} \in V$. By the Zariski openness of the ampleness there exists a neighborhood $W$ of $d\left(y_{0}\right)$ in $D\left(y_{0}\right)$ such that the divisor $Z_{d}$ is ample on $X_{\delta(d)}$ for all $d \in W$. Take any open subset $V_{0} \subseteq V$ on which we can find a holomorphic section $V_{0} \rightarrow W$. Then it is immediate to see that $f$ is projective over $V_{0}$.

q.e.d.

Proposition 2. Let $f: X \rightarrow Y$ be a generically smooth fiber space of compact complex varieties in $\mathcal{C}$. Then any irreducible component of $\mathrm{Div}^{*} X / Y$ is generically locally projective over $Y$.

Proof. Let $D_{k}^{*}$ be any irreducible component of $\mathrm{Div}^{*} X / Y . \quad D_{k}^{*}$ is a compact complex variety in $\mathcal{C}$ and the natural morphism $D_{k}^{*} \rightarrow Y$ is surjective. Let $U \subseteq Y$ be a Zariski open subset over which $f$ is smooth. Let $\mu_{k, U}: D_{k, U}^{*} \rightarrow \mathrm{Pic}_{\gamma} X_{U} / U$ be induced by $\mu_{X_{U} / U}$ where $D_{k}^{*} \cong \operatorname{Div}_{\gamma}^{*} X / Y$. Let $B_{k}:=\mu_{k, U}\left(D_{k, U}^{*}\right) \cong \mathrm{Pic}_{\gamma} X_{U} / U$. Since $\mu_{X_{U} U}$ is projective over any open subset $V \cong U$ over which $f$ admits a holomorphic section (cf. $1.2 \mathrm{c}$ )), it suffices by Lemma 5 to show that the analytic set $B_{k}$ is projective over some open subset of $U$. Let $r_{U}: \widetilde{B}_{k} \rightarrow B_{k}$ be a resolution. Take an open subset $U^{\prime}$ of $U$ such that $\tilde{B}_{k}$ is smooth over $U^{\prime}$. On the other hand, $\left(D_{k, U}^{*}\right)_{y}$ is projective as a compact subspace of $(\operatorname{Div} X / Y)_{y}=\operatorname{Div} X_{y}$ [9]. Hence each fiber of $\tilde{B}_{k, U^{\prime}} \rightarrow U^{\prime}$ is Moishezon. So there exists a relative Albanese map $\varphi: \tilde{B}_{k, U^{\prime}} \rightarrow A=\operatorname{Alb}\left(\tilde{B}_{k, U^{\prime}} / U^{\prime}\right)$ for $\tilde{B}_{k, U^{\prime}} \rightarrow U^{\prime}$ with a smooth structure morphism $\eta: A \rightarrow U^{\prime}$, such that each fiber of $\eta$ is an abelian variety. Then by the universality of the relative Albanese map we have a unique $U^{\prime}$-morphism $h: A \rightarrow$ Pic $X_{U^{\prime}} / U^{\prime}$ such that $h \varphi=i r_{U^{\prime}}$ where $i: B_{k, U^{\prime}} \rightarrow \mathrm{Pic} X_{U^{\prime}} / U^{\prime}$ is the inclusion. Let $\bar{A}=h(A) \subseteq \operatorname{Pic} X_{U^{\prime}} / U^{\prime}$. Then $\bar{A}$ is smooth over $U^{\prime}$ and each fiber is an abelian variety. Hence by Lemma $7 \bar{A} \rightarrow U^{\prime}$ is projective over some open subset $W$ of $U^{\prime}$. As a subspace of $\bar{A}_{U^{\prime}}, B_{k}$ is a fortiori projective over $W$ as was desired.

q. e. d.

2.4. Relative algebraic dimension. a) Let $X$ be a compact complex space and $L$ a line bundle on $X$. Let $\kappa(X, L)$ be the $L$-dimension of $X$ in the sense of litaka (cf. [23]). The following is shown in Lieberman-Sernesi [19]: Let $f$ : $X \rightarrow Y$ be a flat fiber space of complex spaces. Let $L$ be a line bundle on $X$ and $k \geqq 0$ an integer. Then the set $Y_{k}=\left\{y \in Y ; \kappa\left(X_{y}, L_{y}\right) \geqq k\right\}$ is a union of at most 
countably many analytic subvarieties of $Y$.

Lemma 8. Let $f: X \rightarrow Y$ be a generically smooth fiber space of complex spaces. Let $Z$ be a subspace of $X$ of pure codimension 1 . Let $U \subseteq Y$ be a smooth Zariski open subset over which $f$ is smooth and $\left.f\right|_{z}$ is flat. Let $k \geqq 0$ be an integer. Then the set $A_{k}(Z):=\left\{y \in U ; \kappa\left(X_{y},\left[Z_{y}\right]\right) \geqq k\right\}$ is a union of at most countably many analytic subsets of $U$ whose closures in $Y$ are analytic.

Proof. Let $\sigma: \tilde{X} \rightarrow X$ be the blowing up of $X$ with center $Z$ and $\tilde{Z}$ the inverse image of $Z$ in $\tilde{X}$. $\tilde{Z}$ is then a Cartier divisor on $\tilde{X}$ and $\sigma$ is isomorphic over $U$. Let $\tilde{L}=[\tilde{Z}]$ be the line bundle defined by $\tilde{Z}$. Let $\tilde{f}=f \sigma: \tilde{X} \rightarrow Y$. Then take a proper modification $\varphi: Y^{\prime} \rightarrow Y$ such that $\varphi$ is isomorphic on $\varphi^{-1}(U)$ and the strict transform $X^{\prime}$ of $\tilde{X}$ in $\tilde{X} \times_{Y} Y^{\prime}$ is flat over $Y^{\prime}$ (cf. [15]). Let $\phi: X^{\prime} \rightarrow \tilde{X}$ be the natural morphism. Let $L^{\prime}=\phi^{*} \widetilde{L}$. By our construction we may regard $A_{k}(Z) \cong \varphi^{-1}(U) \subseteq Y^{\prime}$. Let $A_{k}\left(L^{\prime}\right):=\left\{y^{\prime} \in Y^{\prime} ; \kappa\left(X_{y^{\prime}}^{\prime}, L_{y^{\prime}}^{\prime}\right) \geqq k\right\}$. Then by the result of Liebermann-Sernesi cited above $A_{k}\left(L^{\prime}\right)$ is a union of at most countably many analytic subvarieties $A_{k}\left(L^{\prime}\right)_{\nu}$ of $Y^{\prime}$ and $A_{k}\left(L^{\prime}\right) \cap \varphi^{-1}(U)=A_{k}(Z)$ with respect to the above identification. It follows that the closure of $A_{k}(Z)$ in $Y$ is a union of those $\varphi\left(A_{k}(L)_{\nu}\right)$ with $A_{k}(L)_{\nu} \cap \varphi^{-1}(U)=\varnothing$.

q. e. d.

b) For any compact complex variety we shall denote by $a(X)$ its algebraic dimension (cf. [23]). When $X$ is nonsingular, then $a(X) \geqq k$ if and only if there exists a line bundle $L$ on $X$ with $\kappa(X, L) \geqq k$.

Proposition 3. Let $f: X \rightarrow Y$ be a generically smooth fiber space of compact complex varieties in $\mathcal{C}$. Let $U \subseteq Y$ be a Zariski open subset over which $f$ is smooth. For any integer $k \geqq 0$ we set $A_{k}:=\left\{y \in U ; a\left(X_{y}\right) \geqq k\right\}$. Then $A_{k}$ is at most $a$ countable union of analytic subsets of $U$ whose closures in $Y$ are analytic.

Proof. Let $\operatorname{Div}^{-} X / Y$ be the closure of $\operatorname{Div} X_{U} / U$ in $D_{X / Y}$ and $Z_{\bar{X} / Y}$ the closure of the universal relative divisor $Z_{X_{U} / U}$ in $\left(\operatorname{Div}^{-} X / Y\right) \times_{Y} X$. Since $Z^{-}$is of pure codimension 1 in $\left(\operatorname{Div}^{-} X / Y\right) \times_{Y} X$, by Lemma 8 the set $B_{k}(U)$ $=\left\{d \in \operatorname{Div} X_{U} / U ; \kappa\left(X_{\delta(d)},\left[Z_{U, d}\right]\right) \geqq k\right\}$ (where $Z=Z_{X / Y}^{*}$ ) is a union of at most countably many analytic subsets $B_{k, \nu}^{0}, \nu \in N$, of $\operatorname{Div} X_{U} / U$ whose closures $B_{k, \nu}$ of $B_{k, \nu}^{0}$ are analytic in $\operatorname{Div}^{-} X / Y$. Since $X \in \mathcal{C}, B_{k, \nu}$ are all compact. Let $\bar{B}_{k, \nu}$ $=\delta\left(B_{k, \nu}\right)$ and $\bar{B}_{k}=\bigcup_{\nu} \bar{B}_{k, \nu}$. Then by the above remark, for $y \in U, a\left(X_{y}\right) \geqq k$ if and only if $y \in \bar{B}_{k}$, i. e., $A_{k}=\bar{B}_{k}$. The proposition follows.

q. e.d.

c) Let $f: X \rightarrow Y$ be as in Proposition 3. Since $A_{k} \supseteq A_{k+1}$ and $A_{0}=U$, there exists a unique maximal $k$ such that $A_{k}=U$. By the above proposition this number $k$ is independent of the choice of $U$ as above.

Definition 2. We shall call $k$ the algebraic dimension of $f$, or the relative algebraic dimension of $X$ over $Y$ and denote it by $a(f) ; a(f)=k$. It follows from the above proposition that $a(f)=k$ if and only if $a\left(X_{y}\right)=k$ for 'general' 
$y \in Y$, i. e., if $y$ is in a complement of at most countably many proper analytic subvarieties of $Y$.

2.5. Relative algebraic reduction.

Definition 3. Let $f: X \rightarrow Y$ be a fiber space of compact complex varieties in c. Then a relative algebraic reduction for $f$ is a commutative diagram

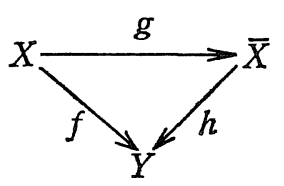

of compact complex varieties in $\mathcal{C}$ where $h$ is a fiber space and $g$ is a meromorphic fiber space such that 1) $a(h)=a(f)$ and 2) $a(h)=\operatorname{dim} h$. We also call the map $g: X \rightarrow \bar{X}$ a relative algebraic reduction of $f$.

Here and in what follows we call a meromorphic map $g: X \rightarrow \bar{X}$ of complex varieties a meromorphic fiber space if $g$ is generically surjective and its general fiber is irreducible.

Proposition 4. Let $f: X \rightarrow Y$ be a generically smooth fiber space of compact complex varieties in $C$. Then there exists a relative algebraic reduction $g: X \rightarrow \bar{X}$ for $f$ such that $\bar{X}$ is generically locally projective over $Y$. In particular if $a(f)=$ $\operatorname{dim} f$, we can always find a bimeromorphic model $f^{\prime}: X^{\prime} \rightarrow Y$ of $f$ which is generically locally projective.

Proof. Let $W \leqq U$ be an open subset on which there exists a holomorphic section $s_{W}$ to $B_{k}(U) \rightarrow U$ where $B_{k}(U)$ is as in the proof of Proposition 3 with $k=a(f)$. The holomorphic line bundle $L_{W}:=\left(i d_{X_{W} \times W} s_{W}\right) *\left[Z_{W}\right]$ on $X_{W}=X_{W} \times_{W} W$ satisfies $\kappa\left(X_{y}, L_{y}\right) \geqq a(f)$ for all $y \in W$ and that the equality holds for 'general' $y$ where $L_{y}=\left.L_{W}\right|_{x_{y}}$. Then after restricting $W$ if necessary, for some sufficiently large $m>0$, the meromorphic $W$-map $X_{W} \rightarrow \boldsymbol{P}\left(f_{*} \mathcal{L}_{W}^{\otimes m}\right)$ associated to the coherent sheaf $f_{*} \mathcal{L}_{W}^{\otimes m}$ has the property that if $\bar{Z}_{W} \subseteq \boldsymbol{P}\left(f_{*} \mathcal{L}_{W}^{\otimes m}\right)$ is the image of the map, then the induced map $\bar{\varphi}_{W} ; X_{W} \rightarrow \bar{Z}_{W}$ is a meromorphic fiber space and $\operatorname{dim} \bar{h}_{W}$ $=a(f)$ where $\bar{h}_{W}: \bar{Z}_{W} \rightarrow W$ is the natural morphism. Let $D_{\alpha}$ be any irreducible component of $\operatorname{Div}^{*} X / Y$ containing $s_{W}(W)$. (Clearly $s_{W}(W) \subseteq \operatorname{Div} * X / Y$ ). We consider the universal meromorphic $Y$-map $X \rightarrow \operatorname{Div} * D_{\alpha} / Y$ associated to the inclusion $Z_{\alpha} \subseteq D_{\alpha} \times_{Y} X$ where $Z_{\alpha}$ is considered to be a relative divisor over a Zariski open subset of $X$ (cf. [5], Lemma 5.1). Let $\bar{X}$ be its image, $g: X \rightarrow \bar{X}$ the resulting meromorphic $Y$-map, and $h: \bar{X} \rightarrow Y$ the natural morphism. Then from the definition of $D_{\alpha}$ together with the construction of $g$ it follows that over $W$ we have a unique meromorphic $W$-map $\eta_{W}: \bar{X}_{W} \rightarrow \bar{Z}_{W}$ such that $\bar{\varphi}_{W}=\eta_{W} g_{W}$. On the other hand, by Proposition $2 \bar{X}$ is generically locally projective over $Y$. 
Hence $\operatorname{dim} h \leqq a(f)$, while we have $a(f)=\operatorname{dim} \bar{h}_{W} \leqq \operatorname{dim} h$. Thus $\operatorname{dim} h=a(f)$ and $\eta_{W}$ must be bimeromorphic. Hence $g_{W}$ is a meromorphic fiber space as well as $\bar{\varphi}_{W}$. Thus $g$ also is a meromorphic fiber space. Hence $g$ is a relative algebraic reduction of $f$.

q. e. d.

Remark 1. Using Chow's lemma [15] we may assume in the final assertion that $X^{\prime}$ is nonsingular and is obtained by a succession of monoidal transformations with nonsingular centers from $X$.

\section{§3. Construction of $\operatorname{Pic}^{*} X / Y$}

Let $f: X \rightarrow Y$ be a generically smooth fiber space of compact complex varieties in $\mathcal{C}$. Let $U$ be a Zariski open subset of $Y$ over which $f$ is smooth. We assume throughout this section that $a(f)=\operatorname{dim} f$.

The purpose of this section is to associate to each such fiber space a complex space Pic* $X / Y$ over $Y$ with a certain 'meromorphic universal property'. It is roughly an 'extension' of the relative Picard variety Pic $X_{U} / U \rightarrow U$ for the smooth morphism $f_{U}: X_{U} \rightarrow U$ to the whole $Y$.

3.1. First we prove two simple lemmas which provide us with the main technique for construction.

Lemma 9. Let $f: X \rightarrow Y$ be a proper surjective morphism of compact complex varieties in $\mathcal{C}$ which is generically smooth. Suppose that there exist Zariski open subsets $V \cong U \subseteq Y$, a proper surjective morphism $g: Z \rightarrow U$ of complex varieties and a $U$-morphism $h: X_{U} \rightarrow Z$ which is a fiber space and is flat over $Z_{V}$. Then there exists a canonical compactification $Z_{V} \subseteq Z^{*}$ of $Z_{V}$ into a compact complex variety $Z^{*}$ in $C$ over $Y$ such that $h_{V}$ extends to a meromorphic $Y$-map $h^{*}: X^{*} \rightarrow Z^{*}$ which is bimeromorphic over $U$ to $h_{U}$.

Proof. Set $W=Z_{V}$. Considering $h_{W}: X_{W} \rightarrow W, X_{W}=\left(X_{U}\right)_{W}$, as a flat family of subspaces of $X$ over $Y$ with respect to the embedding $h_{W} \times_{Y} \iota_{W}: X_{W} \rightarrow W \times_{Y} X$ where $\iota_{W}: X_{W} \rightarrow X$ is the natural inclusion, we get the universal $Y$-morphism $\tau:\left.W \rightarrow D_{X / Y}\right|_{U}$, where $W$ is naturally over $Y$. Then $\tau$ is clearly injective and by [16], Lemma 3, it is an open embedding at each point of $W$. Moreover $\tau$ extends to a meromorphic $Y$-map $\tau^{*}:\left.Z \rightarrow D_{X / Y}\right|_{U}$ (cf. [5]). Hence there exists a unique irreducible component $D_{\alpha}$ of $D_{X / Y, r e d}$ which contains $\tau^{*}(Z)$ as a Zariski open subset of $D_{\alpha, U}$. Let

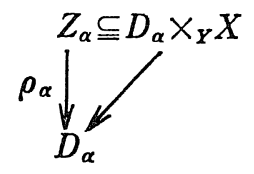

be the universal family restricted to $D_{\alpha}$. By our construction $\rho_{\alpha}$ restricted to 
$\tau(W)$ is naturally isomorphic to $h_{W}$. Therefore the natural map $\pi_{\alpha}: Z_{\alpha} \rightarrow X$ is bimeromorphic, being isomorphic to the inclusion $\iota_{W}: X_{W} \rightarrow X$ over $W=\tau(W)$. Hence it suffices to take $X^{*}=Z_{\alpha}, Z^{*}=D_{\alpha}, h^{*}=\rho_{\alpha}$. Finally the canonicity of the compactification means that if $V^{\prime} \leqq U \subseteq Y$ is another Zariski open subset such that $h_{U}$ is flat over $Z_{V^{\prime}}$, then the resulting complex variety $Z^{* \prime}$ compactifying $Z_{V^{\prime}}$ via the above procedure is canonically isomorphic to the above $Z^{*}$. This is indeed clear from our construction.

Lemma 10. Let $Y$ be a complex variety. Let $V \cong U \subseteq Y$ be Zariski open subsets. Let $X_{1}, X_{1}^{\prime}, X, X^{\prime}$ be reduced complex spaces over $Y$ which are proper over $Y$. Let $\phi: X_{1} \rightarrow X_{1}^{\prime}, h: X_{1} \rightarrow X, h^{\prime}: X_{1}^{\prime} \rightarrow X^{\prime}$ be meromorphic $Y$-maps which are holomorphic over $V$. We assume that $h$ is surjective and each irreducible component of $X$ is mapped surjectively onto $Y$. Let $X_{0}$ and $X_{0}^{\prime}$ be reduced complex spaces over $U$. Suppose that there exist a meromorphic $U$-map $\phi_{0}: X_{0} \rightarrow X_{0}^{\prime}$ and a bimeromorphic U-map $\iota: X_{U} \rightarrow X_{0}$ (resp. $\left.\iota^{\prime}: X_{U}^{\prime} \rightarrow X_{0}^{\prime}\right)$ which is isomorphic over $V$ such that $\phi_{0} h_{U}=\iota^{\prime} h_{U}^{\prime} \psi_{U}$. Then there exists a meromorphic $Y$-map $\bar{\psi}: X \rightarrow X^{\prime}$ such that $\iota^{\prime} \bar{\psi}_{U}=\psi_{0} c$.

Proof. Let $\Gamma \leqq X_{1} \times_{Y} X_{1}^{\prime}$ be the graph of $\phi$. Let $\vec{\Gamma}=h \times_{Y} h^{\prime}(\Gamma) \leqq X \times_{Y} X^{\prime}$. Then since $h$ and $h^{\prime}$ are holomorphic over $V, h$ is surjective, and since $\phi_{0} c h_{U}$ $=\iota^{\prime} h_{U}^{\prime} \psi_{U}, \bar{\Gamma}_{V}:=\bar{\Gamma} \cap\left(X_{V} \times_{V} X_{V}\right)$ coincides with the graph of $\left.\iota^{\prime-1} \psi_{0} \varepsilon\right|_{X_{V}}$. Then the closure $\bar{\Gamma}^{\prime}$ of $\bar{\Gamma}_{V}$ in $X \times_{Y} X^{\prime}$ gives a graph of a meromorphic $Y$-map $\bar{\psi}: X \rightarrow X^{\prime}$ by virtue of our assumption on $X$. Moreover again by the above commutativity, over $U \bar{\Gamma}^{\prime}$ must coincides with the graph of $\iota^{\prime-1} \phi_{0} \iota$.

q. e. d.

Corollary. Let $V \leqq U \leqq Y, X_{0}, X_{0}^{\prime}, X, X^{\prime}, \iota, \iota^{\prime}$, and $\phi_{0}$ be as above. Let $\nu: \tilde{Y} \rightarrow Y$ be a proper surjective morphism of complex varieties. Let $\tilde{X}=X \times_{Y} \tilde{Y}$ and $\tilde{X}^{\prime}=X^{\prime} \times_{Y} \tilde{Y}$. Let $\tilde{U}=\nu^{-1}(U)$ and $\tilde{V}=\nu^{-1}(V)$. If there exists a meromorphic $\tilde{Y}$-map $\phi: \tilde{X} \rightarrow \tilde{X}^{\prime}$ which is bimeromorphic to $\phi_{0} \times_{U} \tilde{U}$ over $\tilde{U}$ and is isomorphic to $\phi_{0} \times_{V} \tilde{V}$ over $\tilde{V}$, the conclusion of the above lemma holds true.

Proof. It suffices to take $X_{1}=\tilde{X}$ and $X_{1}^{\prime}=\tilde{X}^{\prime}$ in the above proposition.

Recall that a proper morphism $f: X \rightarrow Y$ of complex spaces is called Moishezon if it is bimeromorphic to a projective morphism (cf. [6]). We record the following well-known:

Lemma 11. Let $f: X \rightarrow Y$ be a proper morphism of reduced complex spaces. Suppose that there exists a dense Zariski open subset $U \subseteq Y$ such that $X_{y}$ is a complex projective space for any $y \in U$. Then $f$ is Moishezon.

Proof. It suffices to show that for any irreducible component $Y_{i}$ of $Y$ the induced morphism $f_{i}: f^{-1}\left(Y_{i}\right) \rightarrow Y_{i}$ is Moishezon. So we may assume that $Y$ is irreducible. Restricting $U$ we may assume that $U$ is smooth and that $f_{U}: X_{U} \rightarrow U$ is flat and hence is smooth. Let $r: \tilde{X} \rightarrow X$ be a resolution and $\tilde{f}=f r: \tilde{X} \rightarrow Y$. Clearly $\tilde{f}$ satisfies the condition of the lemma. Then the meromorphic $Y$-map 
$\tilde{X} \rightarrow \boldsymbol{P}\left(\tilde{f}_{*} \mathcal{K}_{\tilde{X}}^{-1}\right)$ is bimeromorphic onto its image, where $\mathcal{K}_{\tilde{X}}$ is the canonical sheaf of $\tilde{X}$. Hence $\tilde{f}$, and hence $f$ also, is Moishezon.

q. e. d.

3.2. Let $f: X \rightarrow Y$ and $U \subseteq Y$ be as at the beginning of this section. Then a precise formulation for $\mathrm{Pic}^{*} X / Y$ will now be given in the following:

Definition 4. Let $\left\{\mathrm{Pic}_{\gamma} X_{U} / U\right\}, \gamma \in \Gamma(f)$, be the set of essential components of $\mathrm{Pic} X_{U} / U$ where $\Gamma(f)$ is as in 2.1 a) i). Then we say that $\mathrm{Pic}^{*} X / Y$ exists if the following is true. For any $\gamma \in \Gamma(f)$ there exists a compact complex variety $\mathrm{Pic}_{7}^{*} X / Y$ in $\mathcal{C}$ over $Y$ with the following properties.

1) $\mathrm{Pic}_{\gamma}^{*} X / Y$ and $\mathrm{Pic}_{\gamma} X_{U} / U$ are bimeromorphic to each other over $U$ and are isomorphic over some Zariski open subset $U_{Y}$ of $Y$ with $U_{\gamma} \subseteq U$.

2) For any $\nu: \tilde{Y} \rightarrow Y$ as in 2.1 a) ii), $\mathrm{Pic}_{\gamma}^{*} X / Y \dot{\times}_{Y} \tilde{Y}$ is naturally bimeromorphic over $\tilde{Y}$ to $\operatorname{\amalg Pic}_{\tilde{\gamma}}^{*} \tilde{X} / \tilde{Y}, \tilde{X}=X \dot{\mathrm{X}}_{Y} \tilde{Y}$, where $\tilde{\gamma} \in \Gamma(\nu)^{-1}(\gamma)$.

3) For any $f^{\prime}: X^{\prime} \rightarrow Y$ and $g: X^{\prime} \rightarrow X$ as in 2.1 a) iii) we have a unique meromorphic $Y$-map $g_{\gamma}^{*}: \operatorname{Pic}_{\gamma}^{*} X / Y \rightarrow \operatorname{Pic}_{\gamma^{\prime}}^{*} X^{\prime} / Y, \quad \gamma^{\prime}=\Gamma(g)(\gamma)$, which is bimeromorphic to the natural $U$-morphism $g_{U}^{*}: \mathrm{Pic}_{\gamma} X_{U} / U \rightarrow \mathrm{Pic}_{\gamma^{\prime}} X_{U}^{\prime} / U$.

4) There exists a meromorphic $Y$-map $\mu_{\gamma}^{*}: \operatorname{Div}_{\gamma}^{*} X / Y \rightarrow \operatorname{Pic}_{\gamma}^{*} X / Y$ which is bimeromorphic to $\mu_{\gamma}: \operatorname{Div}_{\gamma} X_{U} / U \rightarrow \operatorname{Pic}_{\gamma} X_{U} / U$ over $U$ (cf. $1.3 \mathrm{c}$ ) v)). Moreover $\mu_{\gamma}^{*}$ is Moishezon (i. e., any of its holomorphic model is Moishezon).

5) There exists a meromorphic $Y$-map $m_{\gamma, \gamma^{\prime}}^{*}: \mathrm{Pic}_{\gamma}^{*} X / Y \dot{\times}_{Y} \mathrm{Pic}_{\gamma^{\prime}}^{*} X / Y \rightarrow \mathrm{Pic}_{\gamma+\gamma^{\prime}}^{*} X / Y$ (resp. $a_{r, \gamma^{\prime}}^{*}: \mathrm{Pic}_{\gamma}^{*} X / Y \dot{\mathrm{X}}_{Y} \mathrm{Pic}_{\gamma^{\prime}}^{*} X / Y \rightarrow \mathrm{Pic}_{\gamma-\gamma^{\prime}}^{*} X / Y$ ) which is bimeromorphic over $U$ to $m_{\gamma, \gamma^{\prime}}: \mathrm{Pic}_{\gamma} X_{U} / U \times{ }_{U} \mathrm{Pic}_{\gamma^{\prime}} X_{U} / U \rightarrow \mathrm{Pic}_{\gamma+\gamma^{\prime}} X_{U} / U \quad$ (resp. $a_{\gamma, \gamma^{\prime}}: \mathrm{Pic}_{\gamma} X_{U} / U \times{ }_{U} \mathrm{Pic}_{\gamma^{\prime}} X_{U} / U$ $\left.\left.\rightarrow \mathrm{Pic}_{\gamma-\gamma^{\prime}} X_{U} / U\right)(\mathrm{cf} .1 .3 \mathrm{c}) \mathrm{v}\right)$ ) such that $\mu_{\gamma+\gamma^{\prime}}^{*} \tilde{m}_{\gamma, \gamma^{\prime}}^{*}=m_{\gamma, \gamma^{\prime}}^{*}\left(\mu_{\gamma}^{*} \times_{Y} \mu_{\gamma^{\prime}}^{*}\right)$ where $\gamma, \gamma^{\prime} \in \Gamma(f)$. Moreover there exists a meromorphic section $Y \rightarrow \mathrm{Pic}_{0}^{*} X / Y$ which is bimeromorphic to the identity section of $\mathrm{Pic}_{0} X_{U} / U \rightarrow U$.

6) Let $\nu: \tilde{Y} \rightarrow Y$ and $\tilde{X}$ be as in 2). Let $\mathscr{I}$ be any coherent analytic sheaf on $\tilde{X}$ which is invertible on $\tilde{X}_{\widetilde{U}}, \tilde{U}=\nu^{-1}(U)$. Let $\tau: \tilde{U} \rightarrow \mathrm{Pic}_{\gamma} X_{U} / U$ be the universal $U$-morphism defined by $\left.\mathscr{F}\right|_{\tilde{X} \tilde{U}}$ for a unique $\gamma \in \Gamma(f)$. Then $\tau$ extends to a unique meromorphic $Y$-map $\tilde{\tau}: \tilde{Y} \rightarrow \mathrm{Pic}_{\gamma}^{*} X / Y$.

7) If $f$ is Moishezon, then the structure morphism $\mathrm{Pic}_{\gamma}^{*} X / Y \rightarrow Y$ also is Moishezon.

8) $\mathrm{Pic}_{\gamma}^{*} X / Y$ is (up to bimeromorphic equivalences over $Y$ ) independent of the choice of $U$, so that in particular the above properties are valid for any $U$ as above.

If $\mathrm{Pic} * X / Y$ exists for $f$ in the sense defined above, we set $\mathrm{Pic}^{*} X / Y$ $=\amalg_{\gamma} \mathrm{Pic}_{\gamma}^{*} X / Y$ which is naturally a complex space over $Y$. In terms of $\mathrm{Pic}^{*} X / Y$ the above properties can informally be stated as follows. 1) $\mathrm{Pic} * X / Y$ is bimeromorphic over $U$ to $\left.\operatorname{Pic} X_{U} / U, 2\right) \operatorname{Pic} * X / Y \times_{Y} \tilde{Y}$ and $\mathrm{Pic} * \tilde{X} / \tilde{Y}$ are naturally bimeromorphic over $Y, 3) g$ induces the natural meromorphic $Y$-map $g^{*}: \operatorname{Pic}^{*} X / Y \rightarrow$ $\left.\mathrm{Pic}^{*} X^{\prime} / Y, 4\right)$ there exists a meromorphic $Y$-map $\mu_{X / Y}^{*}: \operatorname{Div}^{*} X / Y \rightarrow \mathrm{Pic}^{*} X / Y$ which 
is bimeromorphic to $\mu_{X_{U} / U}$ over $\left.U, 5\right)$ there exists a meromorphic $Y$-map $m_{X / Y}^{*}$ (resp. $a_{X / Y}^{*}$ ): $\mathrm{Pic}^{*} X / Y \times{ }_{Y} \mathrm{Pic} * X / Y \rightarrow \mathrm{Pic}^{*} X / Y$ which is bimeromorphic to $m_{X_{U} / U}$ (resp. $a_{X_{U} / U}$ ) over $U, 6$ ) there exists a meromorphic $Y$-map $\tau: \tilde{Y} \rightarrow \operatorname{Pic}^{*} X / Y$ defined by $\mathscr{I}$ which is bimeromorphic to the universal morphism $\tau: \tilde{U} \rightarrow \operatorname{Pic} X_{U} / U$ defined by $\left.\left.\mathscr{I}\right|_{\tilde{X} \tilde{U}}, 8\right) \mathrm{Pic}^{*} X / Y$ is up to bimeromorphic equivalences over $Y$ independent of the choice of $U$ as above.

Then we prove the following:

Theorem 1. Let $f: X \rightarrow Y$ be a generically smooth fiber space of compact complex varieties in $C$ with $a(f)=\operatorname{dim} f$. Then $\mathrm{Pic} * X / Y$ for $f$ exists in the sense of Definition 4.

3.3. Proof of Theorem 1. I. The case where $f$ is generically locally projective.

Case 1. $\gamma$ is s. ample. In this case recall that there exists a Zariski open subset $U_{\gamma} \leqq Y$ such that $\left(\mu_{\gamma}\right)_{U_{\gamma}}: \operatorname{Div}_{\gamma} X_{U_{\gamma}} / U_{\gamma} \rightarrow \operatorname{Pic}_{\gamma} X_{U_{\gamma}} / U_{\gamma}$ is a holomorphic $\boldsymbol{P}^{k}$ bundle for some $k>0$. Recall also that $\operatorname{Div}_{\gamma} X_{U_{\gamma}} / U_{\gamma}$ admits a natural compactification $\operatorname{Div}_{\gamma} X_{U_{\gamma}} / U_{\gamma} \subseteq \operatorname{Div}_{\gamma}^{*} X / Y$ with $\operatorname{Div}_{\gamma}^{*} X / Y$ a compact complex variety in $\mathcal{C}$ over $Y$. Then by Lemma 9 we get a Zariski open embedding $\left(\mathrm{Pic}_{\gamma} X_{U} / U\right)_{U_{\gamma}}=$ $\mathrm{Pic}_{\gamma} X_{U_{\gamma}} / U_{\gamma} \subseteq \mathrm{Pic}_{\gamma}^{*} X / Y$ (where $\mathrm{Pic}_{\gamma}^{*} X / Y$ is a comact complex variety in $\mathcal{C}$ over $Y$ ) such that $\left(\mu_{\gamma}\right)_{U_{\gamma}}:\left(\operatorname{Div}_{\gamma} X_{U} / U\right)_{U_{\gamma}} \rightarrow\left(\operatorname{Pic} X_{U} / U\right)_{U \gamma}$ extends to a meromorphic $Y$-map $\mu_{\gamma}^{*}: \operatorname{Div}_{\gamma}^{*} X / Y \rightarrow \operatorname{Pic}_{\gamma}^{*} X / Y$ which is bimeromorphic to $\mu_{\gamma}$ over $U$. This is our definition of $\mathrm{Pic}_{\gamma}^{*} X / Y$. It is clear that $\mathrm{Pic}_{\gamma}^{*} X / Y$ is independent of the choice of $U$ (cf. Lemma 9). Further since $\left(\mu_{\gamma}\right)_{U_{\gamma}}$ is a $\boldsymbol{P}^{k}$-bundle, $\mu_{\gamma}^{*}$ is Moishezon by Lemma 11. If $f$ is Moishezon, then $\operatorname{Div}_{\gamma}^{*} X / Y \rightarrow Y$ is Moishezon by [6]. Hence by [6], Prop. $1, \mathrm{Pic}_{\gamma}^{*} X / Y$ also is Moishezon over $Y$. Thus we have proved 1), 4), 7) and 8) in Case 1.

2) Consider the following diagram of meromorphic $Y$-maps (cf. $2.1 \mathrm{c})$ ).

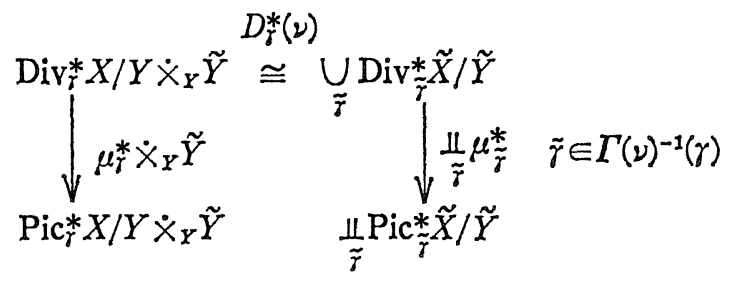

Restricting over $\tilde{U}_{\gamma}:=\nu^{-1}\left(U_{\gamma}\right)$ we get $P_{\gamma}\left(\nu_{U}\right) \mu_{\gamma, \tilde{Y}}^{*}=\left(\amalg \mu_{\tilde{\gamma}}^{*}\right) D_{\gamma}^{*}(\nu)$ where $P_{\gamma}\left(\nu_{U}\right)$ is the isomorphism in $1.3 \mathrm{c}$ ) iii) associated to $\nu_{U}: \tilde{U} \rightarrow U$. Hence 2) follows from Lemma 10.

5) For $m_{\gamma, \gamma^{\prime}}^{*}$ (The case where $\gamma, \gamma^{\prime}$ and $\gamma+\gamma^{\prime}$ are all s. ample.) Write for simplicity $D_{\gamma}^{*}=\operatorname{Div}_{\gamma}^{*} X / Y, P_{\gamma}^{*}=\operatorname{Pic}_{\gamma}^{*} X / Y$ etc. Then consider the following diagram of meromorphic $Y$-maps. 


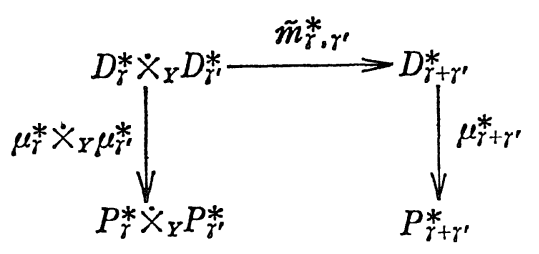

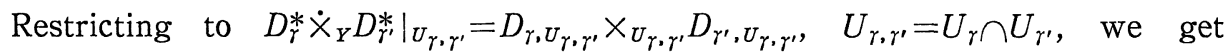
$m_{\gamma, \gamma^{\prime}}\left(\mu_{\gamma}^{*} \dot{\mathrm{X}}_{Y} \mu_{\gamma^{\prime}}^{*}\right)=\mu_{\gamma+\gamma^{\prime}}^{*} \tilde{m}_{\gamma, \gamma^{\prime}}^{*}$. Hence by Lemma 10,5) follows in our special case.

6) First we prove a lemma.

Lemma 12. Let $f: X \rightarrow Y$ and $U$ be as in Theorem 1. Let $\mathscr{F}_{0}$ be a coherent analytic sheaf on $X$ which is invertible over $U$. Let $t: U \rightarrow \operatorname{Pic} X_{U} / U$ be the holomorphic section defined by $\mathscr{F}_{0} \mid x_{U}$. Then $t$ extends to a meromorphic section $t^{*}: Y \rightarrow \mathrm{Pic}_{\gamma}^{*} X / Y$ if $t(U) \leqq \mathrm{Pic}_{\gamma} X_{U} / U$ with $\gamma$ s. ample.

Proof. Let $B=t(U)$ and $C=\mu_{\gamma}^{-1}(U) \leqq \operatorname{Div}_{\gamma} X_{U} / U$. Since $\gamma$ is s. ample, $\mu_{\gamma}(C)=B$. We show that the closure $C^{*}$ of $C$ in $\operatorname{Div}_{\gamma}^{*} X / Y$ is analytic. This would then show that the closure $B^{*}=\mu_{\gamma}^{*}\left(C^{*}\right)$ of $B$ in $\mathrm{Pic}_{\gamma}^{*} X / Y$ is analytic, so that the lemma would follow. To show the analyticity of $C$ take first a suitable proper modification $p: X_{1} \rightarrow X$ so that the strict transform $\mathscr{F}_{1}$ of $\mathscr{F}_{0}$ to $X_{1}$ is invertible [21] and then take a proper modification $\varphi: Y^{\prime} \rightarrow Y$ such that $\varphi$ is isomorphic on $\varphi^{-1}(U)$ and the strict transform $X^{\prime}$ of $X_{1}$ in $X_{1} \times_{Y} Y^{\prime}$ is flat over $Y^{\prime}$ (cf. [15]). Let $\mathscr{I}^{\prime}$ be the pull-back of $\mathscr{I}_{1}$ to $X^{\prime}$. Let $E \rightarrow Y^{\prime}$ be the linear fiber space in the sense of Fischer [4] representing the functor $F:\left(\mathrm{An} / Y^{\prime}\right) \rightarrow(\operatorname{Sets}), \quad F(T)$ $=\Gamma\left(X^{\prime} \times_{Y}, T, \mathscr{F}_{T}^{\prime}\right)$ where $\mathscr{F}_{T}^{\prime}$ is the natural pull-back of $\mathscr{F}^{\prime}$ to $X^{\prime} \times_{Y}, T$. In fact, since $\mathscr{F}^{\prime}$ is invertible, by Schuster [22] $F$ is represented by $p_{+} \mathrm{F}^{\prime}$ where $p_{+}$is the right adjoint functor of the base change functor $p^{+}(T)=X^{\prime} \times_{Y^{\prime}} T$ in the notation of [22], and $F^{\prime}$ is the line bundle corresponding to $\mathscr{F}^{\prime}$. Then the associated projective fiber space $\boldsymbol{P}(E) \rightarrow Y^{\prime}$ is naturally a subspace of $\operatorname{Div}_{\gamma} X^{\prime} / Y^{\prime}$ such that $\boldsymbol{P}(E)_{y}$ is the linear system associated to the line bundle $F_{y}^{\prime}, y \in Y^{\prime}$. Let $q: \operatorname{Div}_{\gamma}^{*} X^{\prime} / Y^{\prime} \rightarrow \operatorname{Div}_{\gamma}^{*} X_{1} / Y^{\prime} \rightarrow \operatorname{Div}_{\gamma}^{*} X / Y$ be the natural bimeromorphic map which is an isomorphism over $U$ if we identify $U$ with $\varphi^{-1}(U)$ via $\varphi$. Then it is easy to see that $C^{*}$ coincides with $q(\boldsymbol{P}(E))$ and hence is analytic. q.e.d.

Returning to the proof of 6) let $\tilde{t}: \tilde{U} \rightarrow \operatorname{Pic}_{\tilde{\gamma}} \tilde{X}_{\tilde{U}} / \tilde{U}$ be the holomorphic section defined by $\left.\mathscr{F}\right|_{\tilde{X} \tilde{U}}$ where $\tilde{\gamma} \in \Gamma(\nu)^{-1}(\gamma)$. Since $\gamma$ is s. ample, $\tilde{\gamma}$ also is s. ample (cf. Lemma 4). Hence by the above lemma applied to $\tilde{f}$ and $\mathscr{I}$ instead of $f$ and $\mathscr{I}_{0}, \tilde{t}$ extends to a meromorphic section $\tilde{t}^{*}: \tilde{Y} \rightarrow \operatorname{Pic} * \tilde{X} / \tilde{Y}$. Then we define $\tilde{\tau}:=P_{\gamma}(\nu) \tilde{t}^{*}$ where $P_{\gamma}(\nu)$ is the natural meromorphic $Y$-map $\operatorname{Pic}_{\tilde{\gamma}}^{*} \tilde{X} / \tilde{Y} \rightarrow \operatorname{Pic}_{\gamma}^{*} X / Y$ (cf. 2)). The desired property is easily checked.

Case 2. The general case. Take s. ample $\alpha, \beta$ with $\alpha-\beta=\gamma$ as in Lemma 6. Let $U_{\alpha}, U_{\beta}$ be as in Case 1 defined respectively for $\alpha$ and $\beta$. Let $W=U_{\alpha} \cap U_{\beta}$. 
Then by our construction in Case 1 we have the natural inclusion $\left(\operatorname{Pic}_{\alpha} X_{U} / U\right)_{W}$ $\times_{W}\left(\operatorname{Pic}_{\beta} X_{U} / U\right)_{W} \leqq \mathrm{Pic}_{\alpha}^{*} X / Y \dot{\times}_{Y} \mathrm{Pic}_{\beta}^{*} X / Y$. Then, since $a_{\alpha \beta}$ is a fiber space, by Lemma 9 we can find a Zariski open embedding $\left(\mathrm{Pic}_{\gamma} X_{U} / U\right)_{W} \rightarrow \mathrm{Pic}_{\gamma}^{*} X / Y$ with $\mathrm{Pic}_{\gamma}^{*} X / Y$ a compact complex variety in $\mathcal{C}$ over $Y$ such that $\left(a_{\alpha \beta}\right)_{W}:\left(\mathrm{Pic}_{\alpha} X_{U} / U\right.$ $\left.\times_{U} \operatorname{Pic}_{\beta} X_{U} / U\right)_{W} \rightarrow\left(\operatorname{Pic}_{\gamma} X_{U} / U\right)_{W}$ extends to a meromorphic $Y$-map $a_{\alpha \beta}^{*}$ : $\operatorname{Pic}_{\alpha}^{*} X / Y$ $\dot{X}_{Y} \mathrm{Pic}_{\beta}^{*} X / Y \rightarrow \mathrm{Pic}_{\gamma}^{*} X / Y$ which is bimeromorphic to $a_{\alpha \beta}$ over $U$. If $f$ is Moishezon, then $\operatorname{Pic}_{\alpha}^{*} X / Y$ and $\operatorname{Pic}_{\beta}^{*} X / Y$ are Moishezon over $Y$ by Case 1 , and hence $\operatorname{Pic}_{\gamma}^{*} X / Y$ also is Moishezon over $Y, a_{\alpha \beta}^{*}$ being surjective. Moreover if $\gamma=0$ and $\alpha=\beta$, then $a_{\alpha \alpha}^{*}\left(\Delta_{\alpha}\right) \subseteq \mathrm{Pic}_{0}^{*} X / Y \quad\left(\Delta_{\alpha}=\right.$ the diagonal in $\left.\mathrm{Pic}_{\alpha}^{*} X / Y \dot{\mathrm{X}}_{Y} \mathrm{Pic}_{\alpha}^{*} X / Y\right)$ defines the desired extension of the identity section of $\operatorname{Pic}_{0} X_{U} / U \rightarrow U$. Thus we have proved the existence of $\operatorname{Pic}_{\gamma}^{*} X / Y$ satisfying 1) (set $U_{r}=W$ ), part of 5) and 7). Before proceeding, however, it is reasonable to check that the above cunstruction is independent of the chosen $\alpha$ and $\beta$.

Lemma 13. Write $\operatorname{Pic}_{\gamma}^{*} X / Y=\operatorname{Pic}_{\gamma}^{*} X / Y_{(\alpha, \beta)}$ for the $\operatorname{Pic}_{\gamma}^{*} X / Y$ constructed above. Then $\operatorname{Pic}_{\gamma}^{*} X / Y_{(\alpha, \beta)}$ are naturally bimeromorphic to one another over $Y$ for various choices of s. ample $\alpha, \beta$ with $\alpha-\beta=\gamma$.

Proof. For give $\alpha, \beta$ we take any s. ample $\delta$ such that both $\alpha+\delta$ and $\beta+\delta$ are s. ample and that $a_{\alpha+\delta, \beta+\delta}$ is a fiber space (cf. Lemma 6). We show that $\operatorname{Pic}_{\gamma}^{*} X / Y_{(\alpha, \beta)}$ and $\operatorname{Pic}_{\gamma}^{*} X / Y_{(\alpha+\delta, \beta+\delta)}$ are bimeromorphically equivalent over $Y$. (The general case follows from this special case readily.) By 2) in Case 1, together with Corollary to Lemma 10 , replacing $f$ by $f \dot{X}_{Y} i d_{\mathrm{Pic}_{\delta}^{*} X / Y}$ (id=identity) if necessary we may assume from the beginning that there exists a meromorphic section $s: Y \rightarrow \operatorname{Pic}_{\delta}^{*} X / Y$. Let $s(Y)=Y^{\prime}$. Let $c_{\alpha}^{*}: \operatorname{Pic}_{\alpha}^{*} X / Y \rightarrow \operatorname{Pic}_{\alpha+\delta}^{*} X / Y$ be the bimeromorphic $Y$-map which is by definition the composite of the bimeromorphic $Y$-map $i d \dot{\times}_{Y} s b_{\alpha}^{*}: \mathrm{Pic}_{\alpha}^{*} X / Y \rightarrow \operatorname{Pic}_{\alpha}^{*} X / Y \dot{\times}_{Y} Y^{\prime}\left(b_{\alpha}^{*}: \mathrm{Pic}_{\alpha}^{*} X / Y \rightarrow Y\right.$ being the natural map) and the restriction $\left(m_{\alpha, \delta}^{*}\right)_{Y^{\prime}}: \mathrm{Pic}_{\alpha}^{*} X / Y \dot{\mathrm{X}}_{Y} Y^{\prime} \rightarrow \mathrm{Pic}_{\alpha+\delta}^{*} X / Y$ of $m_{\alpha, \delta}^{*}$ to $\operatorname{Pic}_{\alpha}^{*} X / Y \dot{\times}_{Y} Y^{\prime}$ where $m_{\alpha, \delta}^{*}$ is as in 5) in Case 1. Define $c_{\beta}^{*}: \operatorname{Pic}_{\beta}^{*} X / Y \rightarrow \operatorname{Pic}_{\beta+\delta}^{*} X / Y$ similarly. Then over $U a_{\alpha \beta}=a_{\alpha+\delta, \beta+\delta}\left(c_{\alpha}^{*} \dot{X}_{Y} c_{\beta}^{*}\right)$ as a $U$-morphism $\mathrm{Pic}_{a} X_{U} / U$ $\times_{U} \mathrm{Pic}_{\beta} X_{U} / U \rightarrow \mathrm{Pic}_{\gamma} X_{U} / U$ where $\left(c_{\alpha}^{*} \dot{X}_{X} c_{\beta}^{*}\right)_{U}$ gives a $U$-isomorphism of $\mathrm{Pic}_{a} X_{U} / U$ $\times_{U} \mathrm{Pic}_{\beta} X_{U} / U$ and $\mathrm{Pic}_{\alpha+o} X_{U} / U \times_{U} \mathrm{Pic}_{\beta+\delta} X_{U} / U$. Hence by Lemma 10 the identity $\operatorname{Pic}_{\gamma}^{*} X /\left.Y_{(\alpha, \beta)}\right|_{W}=\operatorname{Pic}_{\gamma} X_{W} / W=\operatorname{Pic}_{\gamma}^{*} X /\left.Y_{(a+\tilde{o}, \beta+\delta)}\right|_{W}$ extends to a desired bimeromorphic equivalence of $\operatorname{Pic}_{\gamma}^{*} X / Y_{(\alpha, \beta)}$ and $\left.\operatorname{Pic}_{\gamma}^{*} X / Y_{(\alpha+\delta, \beta+\delta)}{ }^{*}\right)$

2) Consider the following diagram

$$
\begin{aligned}
& \left(\operatorname{Pic}_{\alpha}^{*} X / Y \dot{\times}_{Y} \tilde{Y}\right) \dot{X}_{\tilde{Y}}\left(\operatorname{Pic}_{\beta}^{*} X / Y \dot{X}_{Y} \tilde{Y}\right) \stackrel{h}{\longrightarrow}\left(\frac{\Perp}{\tilde{\alpha}} \operatorname{Pic}_{\tilde{\alpha}}^{*} \tilde{X} / \tilde{Y}\right) \dot{X}_{\tilde{Y}}\left(\frac{\Perp}{\tilde{\beta}} \operatorname{Pic}_{\tilde{\beta}}^{*} \tilde{X} / \tilde{Y}\right)
\end{aligned}
$$

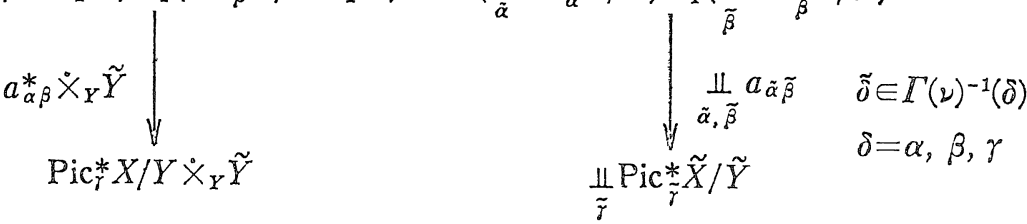

$\left.{ }^{*}\right)$ Here $W=U_{a} \cap U_{\beta} \cap U_{a+\delta} \cap U_{\beta+\delta}$. 
where $h$ is the bimeromorphic map given in Case 1. Then over $\tilde{U}_{\gamma}:=\nu^{-1}\left(U_{\gamma}\right)$ $\left(U_{\gamma}=U_{\alpha} \cap U_{\beta}\right)$ the natural isomorphism $P_{\gamma}\left(\nu_{U_{\gamma}}\right):\left(\mathrm{Pic}_{\gamma}^{*} X / Y \dot{\times}_{Y} \tilde{Y}\right) \widetilde{U}_{\gamma}=\left(\mathrm{Pic}_{\gamma} X_{U_{\gamma}} / U_{\gamma}\right)$ $\times_{U_{\gamma}} \tilde{U}_{\gamma} \cong \prod_{\tilde{\gamma}} \operatorname{Pic} \tilde{\gamma} \tilde{X}_{\tilde{U} \tilde{\gamma}} / \tilde{U}_{\tilde{\gamma}}=\left(\operatorname{II}_{\tilde{\gamma}} \mathrm{Pic}_{\tilde{\gamma}}^{*} \tilde{X} / \tilde{Y}\right)_{\tilde{U}_{\gamma}}$ induced by $\nu_{U_{\gamma}}: \tilde{U}_{\gamma} \rightarrow U_{\gamma}$ (cf. $\left.\left.\left.1.3 \mathrm{c}\right) \mathrm{iii}\right)\right)$ makes the above diagram commutative. Hence 2) follows from Lemma 10.

3) i) Assume that $\gamma$ is s. ample. Consider the diagram

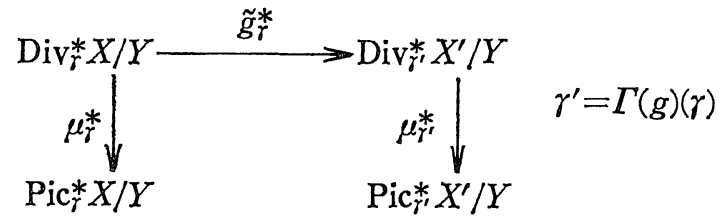

Over $W=U_{\gamma} \cap U_{\gamma^{\prime}}$ the natural morphism $g_{\gamma, W}^{*}:\left(\operatorname{Pic}_{\gamma}^{*} X / Y\right)_{W}=\operatorname{Pic}_{\gamma} X_{W} / W \rightarrow \operatorname{Pic}_{\gamma^{\prime}} X_{W}^{\prime} / W$ $=\left(\mathrm{Pic}_{\gamma^{\prime}}^{*} X^{\prime} / Y\right)_{W}$ makes the above diagram commutative. Hence 3 ) follows from Lemma 10.

ii) In the general case we observe the following diagram

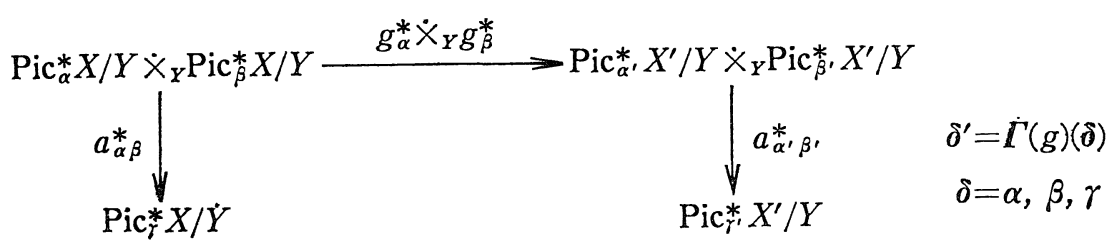

Then by the same argument as in i), 3) follows.

4) Write $\gamma=\alpha-\beta$ with $\alpha, \beta$ s. ample. By 2), 2.1 c) i) and Corollary to Lemma 10, replacing $f$ by $f \dot{\times}_{Y} i d_{\operatorname{Div}_{\beta}^{*} X / Y}$ if necessary, we may assume that $\operatorname{Div}_{\beta}^{*} X / Y \rightarrow Y$ admits a meromorphic section $\tilde{s}_{\beta}$. This induces a meromorphic section $s_{\beta}$ of $\operatorname{Pic}_{\beta}^{*} X / Y \rightarrow Y$ via $\mu_{\beta}^{*}$ which exists by Case 1 . Identifying $\operatorname{Div}_{\gamma}^{*} X / Y$ and $\operatorname{Pic}_{\gamma}^{*} X / Y$ with $\operatorname{Div}_{\gamma}^{*} X / Y \dot{\times}_{Y} \tilde{s}_{\beta}(Y)$ and $\operatorname{Pic}_{\gamma}^{*} X / Y \dot{\times}_{Y} s_{\beta}(Y)$ respectively up to bimeromorphic equivalences over $Y$, these sections define meromorphic maps $\tilde{c}: \operatorname{Div}_{\gamma}^{*} X / Y \rightarrow \operatorname{Div}_{\alpha}^{*} X / Y$ and $c: \operatorname{Pic}_{\gamma}^{*} X / Y \rightarrow \operatorname{Pic}_{\alpha}^{*} X / Y$ respectively with $c$ bimeromorphic such that $c \mu_{\gamma}=\mu_{\alpha}^{*} \tilde{c}$ over $U_{\gamma}$ as a meromorphic map. Then $\mu_{\gamma}^{*}$ is given by $\mu_{\gamma}^{*}=c^{-1} \mu_{\beta}^{*} \tilde{c}$. The last assertion then follows from that for $\mu_{\beta}^{*}$ by the generic injectivity of $\tilde{c}$.

5) Write $\gamma=\alpha-\beta$ and $\gamma^{\prime}=\alpha^{\prime}-\beta^{\prime}$ with $\alpha, \beta, \alpha^{\prime}, \beta^{\prime}$ s. ample. Taking these suitably we may assume that $\alpha+\alpha^{\prime}$ and $\beta+\beta^{\prime}$ are also s. ample. Write for simplicity $D_{\alpha}^{*}=\operatorname{Div}_{\alpha}^{*} X / Y, P_{\alpha}^{*}=\operatorname{Pic}_{\alpha}^{*} X / Y$ etc. Then consider the following diagram of meromorphic $Y$-maps. 


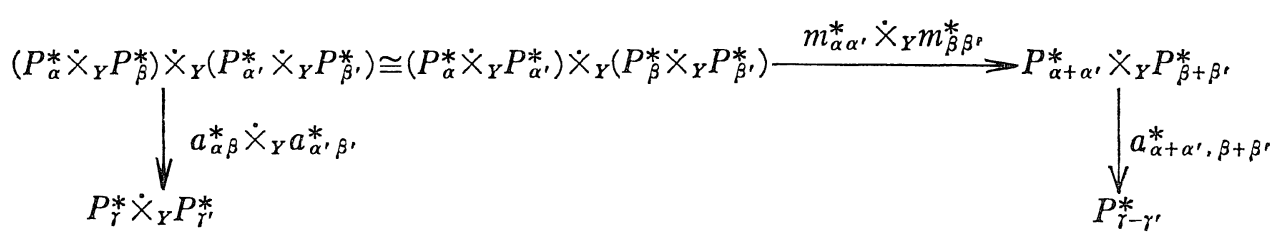

Since over a small Zariski open subset we get a morphism $a_{\gamma, \gamma^{\prime}}: P_{\gamma}^{*} \dot{\mathrm{X}}_{Y} P_{\gamma^{\prime}}^{*} \rightarrow P_{\gamma-\gamma^{\prime}}^{*}$ making the above diagram commutative 5) follows from Lemma 10 . The proof for $m_{r r}^{*}$ is similar.

6) Let $\gamma=\alpha-\beta$ with $\alpha$ and $\beta$ s. ample. i) Suppose first that there exists a holomorphic section $s: Y \rightarrow \operatorname{Div}_{\beta}^{*} X / Y$. Consider the coherent analytic sheaf $\mathcal{L}_{\beta}:=\left(i d_{X} \dot{X}_{Y} S \nu\right) *\left(\mathscr{H}_{o m_{\mathcal{O}}}\left(\mathcal{J}_{\beta}, \mathcal{O}\right)\right), \mathcal{O}=\mathcal{O}_{X} \dot{\times}_{Y} \operatorname{Div}_{\beta}^{*} X / Y$, on $\tilde{X}$ where $\mathcal{G}_{\beta}$ is the ideal

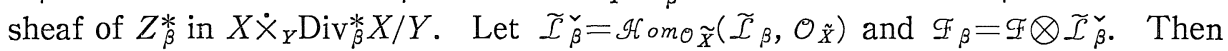
$\left.\mathscr{F}_{\beta}\right|_{\tilde{X} \widetilde{U}}$ is invertible and induces the universal $U$-morphism $\tau^{\prime}: \tilde{U} \rightarrow \operatorname{Pic} X_{U} / U$, with $\tau^{\prime}(\tilde{U}) \subseteq \operatorname{Pic}_{\alpha} X_{U} / U$, as follows from the relation $\alpha=\beta+\gamma$. By what we have proved in Case 1 there exists a meromorphic $Y$-map $\tau^{*}: \tilde{Y} \rightarrow \operatorname{Pic}_{a}^{*} X / Y$ which is bimeromorphic to $\tau^{\prime}$ on $U$. On the other hand, the surjective meromorphic $Y$ map $a_{\alpha \beta}^{*}: \mathrm{Pic}_{\alpha}^{*} X / Y \times_{Y} \mathrm{Pic}_{\beta}^{*} X / Y \rightarrow \mathrm{Pic}_{\gamma}^{*} X / Y$ restricted to $\operatorname{Pic}_{\alpha}^{*} X / Y \dot{\times}_{Y} s(Y)$ defines a bimeromorphic $Y$-map $\varphi_{\alpha \gamma}: \operatorname{Pic}_{\alpha}^{*} X / Y \rightarrow \operatorname{Pic}_{\gamma}^{*} X / Y$. Further we infer readily that $\tau^{\prime}=\left.\varphi_{\alpha \gamma}\right|_{U} \cdot \tau$. Hence $\tau^{*}=\varphi_{\alpha \gamma}^{*-1} \tau^{\prime *}$ is a desired meromorphic map.

ii) Next we consider the general case. For simplicity of notation, however, we consider only the case where $\tilde{Y}=Y$ and leave the general case to the reader. Let $\xi: Y_{1} \rightarrow Y$ be the natural proper surjective morphism where $Y_{1}=\operatorname{Div}_{\beta}^{*} X / Y$, so that $Y_{1} \dot{\times}_{Y} \operatorname{Div}_{\beta}^{*} X / Y \rightarrow Y_{1}$ admits a holomorphic section. Let $U_{1}=\xi^{-1}(U)$ and $X_{1}=X \dot{\times}_{Y} Y_{1}$. Let $\mathscr{F}_{1}$ be the pull-back of $\mathscr{I}$ to $X_{1}$ so that $\left.\mathscr{I}_{1}\right|_{X \times U_{U_{1}}}$ defines a holomorphic section $\tau_{1}: U_{1} \rightarrow \operatorname{Pic}\left(X_{1, U_{1}} / U_{1}\right)$. Let $p_{2}$ : Pic* $X_{1} / Y_{1} \rightarrow \operatorname{Pic}^{*} X / Y$ be the meromorphic $Y$-map which is bimeromorphic over $U_{1}$ to the natural projection $p_{2}: \operatorname{Pic}\left(X_{1, U_{1}} / U_{1}\right) \rightarrow \operatorname{Pic} X_{U} / U$ (cf. 2)). Then we have $p_{2} \tau_{1}=\tau\left(\left.\xi\right|_{U_{1}}\right)$. Since there exists a meromorphic $Y_{1}$-map $\tau_{1}^{*}: Y_{1} \rightarrow \mathrm{Pic}^{*} X_{1} / Y_{1}$ which is bimeromorphic to $\tau_{1}$ over $U_{1}$ by i), it follows that there exists also a meromorphic $Y$-map $\tau^{*}: Y$ $\rightarrow$ Pic* $X / Y$ which is bimeromorphic to $\tau$ over $U$ by Lemma 10 . From this 6 ) follows.

II. The general case. By Proposition 4 (cf. Remark 1) we can find a bimeromorphic $Y$-morphism $\sigma: X^{\prime} \rightarrow X$ of compact complex varieties such that the induced morphism $f^{\prime}=f \sigma: X^{\prime} \rightarrow Y$ is generically smooth and generically locally projective. Then $\sigma$ induces a natural injection $\Gamma(\sigma): \Gamma(f) \rightarrow \Gamma\left(f^{\prime}\right)$ such that $\mathrm{Pic}_{\gamma} X_{U} / U \cong \mathrm{Pic}_{\gamma^{\prime}} X_{U}^{\prime} / U, \gamma^{\prime}=\Gamma(\sigma) \gamma$, over any Zariski open subset $U$ of $Y$ over which both $f$ and $f^{\prime}$ are smooth. Then we set $\operatorname{Pic}_{\gamma}^{*} X / Y=\operatorname{Pic}_{\gamma^{\prime}}^{*} X^{\prime} / Y$ where $\mathrm{Pic}_{\gamma^{\prime}}^{*} X^{\prime} / Y$ is constructed in I. The independence of $\mathrm{Pic}_{\gamma}^{*} X / Y$ (up to bimeromorphic equivalences over $Y$ ) of the choice of $f^{\prime}$ as above follows immediately from the property 3 ) in I together with the following fact; given two bimeromorphic fiber 
spaces $f_{i}: X_{i} \rightarrow Y, i=1,2$, we can always find another fiber space $f_{3}: X_{3} \rightarrow Y$ which is generically locally projective and which dominates holomorphically and bimeromorphically both $f_{1}$ and $f_{2}$.

Form the definition the properties 1), 2), 3), 5), 7), 8) follow immediately from the case I. 4) Let $\tilde{g}^{*}: \operatorname{Div}_{\gamma}^{*} X / Y \rightarrow \operatorname{Div}_{\gamma^{\prime}}^{*} X^{\prime} / Y$ be induced by $g$ with $X^{\prime}$ as above (cf. $2.1 \mathrm{c})$ ii)). Then we have only to set $\mu_{\gamma^{\prime}}^{*}=\mu_{\gamma}^{*} g^{*}$. 6) Let $\tilde{\sigma}:=\sigma \dot{\times}_{Y} \tilde{Y}$ : $X^{\prime} \dot{X}_{Y} \tilde{Y} \rightarrow \tilde{X}=X \dot{\times}_{Y} \tilde{Y}$. Then we have only to define $\tilde{\tau}: \tilde{Y} \rightarrow \operatorname{Pic}_{\gamma^{\prime}}^{*} X^{\prime} / Y=\operatorname{Pic}_{\gamma}^{*} X / Y$ to be the universal meromophic map defined by $\tilde{\sigma}^{*} F$.

3.4. Meromorphic Poincare sheaf. a) In the proof of the next proposition and also in Section 4 we adopt the following convention. Let $f: X \rightarrow Y$ and $f^{\prime}$ : $X^{\prime} \rightarrow Y$ be proper morphisms of complex spaces. Let $\varphi: X \rightarrow X^{\prime}$ be a meromorphic $Y$-map. Let $\Gamma \leqq X \times_{Y} X^{\prime}$ be the graph of $\varphi$ and let $q: \Gamma \rightarrow X$ and $q^{\prime}: \Gamma \rightarrow X^{\prime}$ be the natural projections. Let $\mathscr{F}$ be a coherent analytic sheaf on $X$. Then we write for simplicity $\varphi_{*} \mathscr{F}=q_{*}^{\prime} q^{*} \mathscr{F}$.

b) Let $f: X \rightarrow Y$ be a generically smooth fiber space of compact complex varieties with $a(f)=\operatorname{dim} f$.

Proposition 5. Let $W \subseteq Y$ be an open subset. Suppose that $f$ admits a meromorphic section $s: W \rightarrow X_{W}$. Let $U_{0}$ be a Zariski open subset of $W$ on which $s$ is defined. Then there exists a coherent analytic sheaf $\mathcal{L}$ on $X_{W} \dot{\mathrm{X}}_{W}\left(\mathrm{Pic}^{*} X / Y\right)_{W}$ such that for any $\gamma \in \Gamma(f)$ if $\mathcal{L}_{\gamma}$ is the restriction of $\mathcal{L}$ to $X_{W} \dot{X}_{W}\left(\mathrm{Pic}_{\gamma}^{*} X / Y\right)_{W}$, then on $X_{V_{\gamma}} \dot{\mathrm{X}}_{V_{\gamma}} \mathrm{Pic}_{\gamma}\left(X_{V_{\gamma}} / V_{\gamma}\right)$ where $V_{r}=U_{r} \cap U_{0}, \mathcal{L}_{\gamma}$ is invertible and coincides with the relative Poincare sheaf for the smooth map $f_{V_{r}}$ associated to the section $\left.s\right|_{v_{\gamma}}$

Proof. First we assume that $f$ is generically locally projective. For simplicity of notation we only consider the case $W=Y$. (The proof is completely the same in the general case.) It suffices to construct $\mathcal{L}_{\gamma}$ on each $X \dot{\times}_{Y} P_{\gamma}^{*}$ with the desired property. For simplicity we write $D_{\gamma}^{*}=\operatorname{Div}_{\gamma}^{*} X / Y, P_{\gamma}^{*}=\operatorname{Pic}_{\gamma}^{*} X / Y$ etc.

Case 1. Assume that $\gamma$ is s. ample, so that $\mu_{\gamma}^{*}: D_{\gamma}^{*} \rightarrow P_{\gamma}^{*}$ is a holomorphic $\mathbb{P}^{k_{-}}$ bundle over $\left(P_{\gamma}^{*}\right)_{U_{\gamma}}$ for some $k>0$. Let $Z_{\gamma}^{*} \cong X \dot{\times}_{Y} D_{\gamma}^{*}$ be the associated meromorphic universal divisor. Let $\mathcal{I}_{\gamma}$ be the ideal sheaf of $Z_{\gamma}^{*}$ and let $\mathscr{F}_{\gamma}$ $=\mathscr{I}_{\text {omo }_{\gamma}}\left(\mathcal{G}_{\gamma}, \mathcal{O}_{\gamma}\right)$ where $\mathcal{O}_{\gamma}=\mathcal{O}_{X} \dot{\times}_{Y} D_{\gamma}^{*}$. Set $S=s(Y)$ and let $q: X \dot{\times}_{Y} D_{\gamma}^{*} \rightarrow D_{\gamma}^{*}$ be the natural projection. Let $Z_{\gamma^{\prime}}^{*}=q^{-1} q\left(\left(S \dot{\times}_{Y} D_{\gamma}^{*}\right) \cap Z_{\gamma}^{*}\right) \subseteq X \dot{\times}_{Y} D_{\gamma}^{*} . \quad Z_{\gamma}^{* \prime}$ is a relative divisor over $D_{\gamma, V}^{*}, \gamma$ being s. ample, and in fact $\left(Z_{\gamma}^{* \prime}\right)_{u}=Z_{\gamma}^{*} \cap\left(s(u) \times D_{\gamma}^{*}\right)$ for $u \in V_{r}$. Let $\mathcal{G}_{\gamma}^{\prime}$ be the ideal sheaf of $Z_{\gamma}^{* \prime}$, and set $\mathcal{E}_{\gamma}=\mathscr{I}_{\gamma} \otimes_{\mathcal{O}_{\gamma}} \mathcal{I}_{\gamma}^{\prime}$. Then $\mathcal{L}_{\gamma}$ : $=\left(i d_{X} \dot{X}_{Y} \ell_{l}\right)_{*}\left(\mathcal{E}_{7}\right)$ is a coherent analytic sheaf on $X \dot{\times}_{Y} P_{\gamma}^{*}$ (cf. a)). We claim that this $\mathcal{L}_{\gamma}$ has the desired property. In fact, by the definition $\varepsilon_{\gamma}$ is invertible on $\left(X \dot{\times}_{Y} D_{\gamma}^{*}\right)_{U_{\gamma}}$ and trivial when restricted to each fiber $\{x\} \times D_{\gamma, p}^{*},(x, p) \in X \dot{\times}_{Y} P_{\gamma}^{*}$, of $i d_{Y} \dot{X}_{Y} \mu_{\gamma}^{*}$ over $U_{\gamma}$. Hence, $i d_{X} \dot{X}_{Y} \mu_{r}^{*}$ being a holomorphic $\mathbb{P}^{k}$-bundle over $U_{\gamma}, \mathcal{L}_{\gamma}$ also is invertible over $U_{\gamma}$. Further since $H^{1}\left(D_{\gamma, p}^{*}, \mathcal{O}_{D_{\gamma}^{*}, p}\right)=0, \mathcal{E}_{\ddot{\gamma}}$ is cohomologically flat (in dimension zero) with respect to $\left.i d_{X} \dot{\times}_{Y} \mu_{\gamma}^{*}\right|_{\left(X \vee_{Y} D_{i}^{*}\right)_{i}}$ (cf. [1]). 


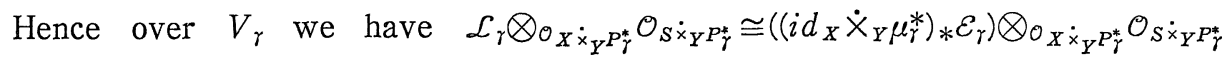
$\cong\left(i d_{S} \dot{\times}_{Y} \mu_{\gamma}^{*}\right)_{*}\left(\mathcal{E}_{\gamma} \otimes_{\mathcal{O}_{X} \dot{\times}_{Y} D_{\gamma}^{*}} \mathcal{O}_{S \dot{x}_{Y} D_{\gamma}^{*}} \cong\left(i d_{S} \dot{\times}_{Y} \mu_{\gamma}^{*}\right)_{*} \mathcal{O}_{S} \dot{x}_{Y} D_{\gamma}^{*} \cong \mathcal{O}_{S \dot{x}_{Y} P_{\gamma}^{*}}\right.$. Thus it suffices to show that for $y \in V_{\gamma}$ the restriction $\mathcal{L}_{\gamma, y}$ of $\mathcal{L}_{\gamma}$ to $\left(X \dot{\mathrm{X}}_{Y} P_{\gamma}^{*}\right)_{y} \cong X_{y} \times P_{\gamma, y}^{*}$ is the normalized Poincare sheaf (restricted) on $X_{y} \times P_{r, y}^{*}$ associated to the base point $s(y) \in X_{y} . \quad\left(P_{\gamma, y}^{*}\right.$ is a union of connected components of Pic $X_{y}$. $)$ In fact, by the cohomological flatness of $\mathcal{E}_{\gamma}, \mathcal{L}_{\gamma, y}=\left(i d_{X} \times \mu_{\gamma, y}^{*}\right)_{*}\left(\mathcal{E}_{\gamma} \otimes_{\mathcal{O}} \mathcal{O}_{X_{y} \times D_{\gamma, y}^{*}}\right)$, $\mathcal{O}=\mathcal{O}_{X_{y} \times D_{\gamma, y}^{*}}$ for $y \in V_{\gamma}$ where $\mu_{\gamma, y}^{*}: D_{\gamma, y}^{*} \rightarrow P_{\gamma, y}^{*}$, and then the result follows from the absolute case (Lemma 1).

Case 2. Write $\gamma=\alpha-\beta$ with $\alpha, \beta$ s. ample as in Lemma 6. Let $q_{\alpha}$ : $P_{\alpha}^{*} \dot{X}_{Y} P_{\beta}^{*} \rightarrow P_{\alpha}^{*}, \quad q_{\beta}: P_{\alpha}^{*} \dot{X}_{Y} P_{\beta}^{*} \rightarrow P_{\beta}^{*}$ be the natural projections and let $\widetilde{\mathcal{L}}_{\alpha}$ $=\left(i d_{X} \times_{Y} q_{\alpha}\right)^{*} \mathcal{L}_{\alpha}, \widetilde{\mathcal{L}}_{\beta}=\left(i d_{X} \times_{Y} q_{\beta}\right)^{*} \mathcal{L}_{\beta}$ where $\mathcal{L}_{\alpha}$ and $\mathcal{L}_{\beta}$ are constructed in Case 1 for $\gamma=\alpha$ and $\beta$ respectively. Then we set $\mathcal{L}_{\gamma}=\left(i d_{X} \dot{\times}_{Y} a_{\alpha \beta}^{*}\right)_{*}\left(\widetilde{\mathcal{L}}_{a} \otimes_{\odot} \widetilde{\mathcal{L}}_{\beta}^{r}\right)$ where $\mathcal{O}=\mathcal{O}_{X} \dot{\times}_{Y} P_{\alpha}^{*} \dot{x}_{Y} P_{\beta}^{*}$ and $a_{\alpha \beta}^{*}: P_{\alpha}^{*} \dot{x}_{Y} P_{\beta}^{*} \rightarrow P_{\gamma}^{*}$ is as in Case 2 of the construction of $P_{\gamma}^{*}$ (cf. a)). Then by $1.1 \mathrm{~d}) \mathcal{L}_{\gamma}$ is invertible over $U_{\gamma}$ and is the relative Poincare sheaf over $V_{\gamma}$ associated to $\left.s\right|_{V_{\gamma}}$, as was desired.

In the general case, take a proper modification $\sigma: X^{\prime} \rightarrow X$ as in $3.3 \mathrm{II}$. Let $\mathcal{L}_{\gamma^{\prime}}, \gamma^{\prime}=\Gamma(\sigma) \gamma$, be an extension of the relative Poincaré sheaf on $X^{\prime} \dot{\times}_{Y} \mathrm{Pic}_{\gamma}^{*} X^{\prime} / Y$ constructed above for $X^{\prime}$ and $\sigma^{-1}$ s. Let $r^{\prime}: C^{\prime} \rightarrow X^{\prime} \dot{\times}_{Y} \mathrm{Pic}_{\gamma^{\prime}}^{*} X^{\prime} / Y, r: C \rightarrow X \dot{\mathrm{X}}_{Y} \mathrm{Pic} \mathrm{C}_{\gamma}^{*} X / Y$ be resolutions of respective spaces such that the strict transform $\mathcal{L}_{C^{\prime}}$ of $\mathcal{L}_{\gamma^{\prime}}$ on $C^{\prime}$ is invertible and there exists a morphism $\Sigma: C^{\prime} \rightarrow C$ such that $r \Sigma$ $=\left(\sigma \times_{Y} i d_{\mathrm{Pic}_{\gamma}^{*} X^{\prime} / Y}\right) r^{\prime}$. Let $L_{C^{\prime}}$ be the line bundle corresponding to $\mathcal{L}_{C^{\prime}}$. Let $L_{C}=\Sigma_{*} L_{C^{\prime}}$ be the direct image of $L_{C^{\prime}}$ as a line bundle [7]. Then we set $\mathcal{L}_{\gamma}=r_{*} \mathcal{L}_{C}$, and it is easy to see that $\mathcal{L}_{\gamma}$ meet the requirement of the proposition.

$$
\text { q. e. d. }
$$

We call any $\mathcal{L}$ with the property of the above proposition a meromorphic relative Poincaré sheaf associated to s.

\section{§4. Relative Albanese Variety}

\subsection{Statement of the theorem.}

Definition 5. Let $f: X \rightarrow Y$ be a generically smooth fiber space of compact complex varieties in $\mathcal{C}$. Then a relative Albanese map for $f$ in $\mathcal{C}$ is a commutative diagram

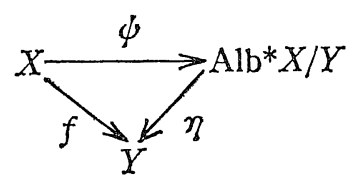

where $\mathrm{Alb}^{*} X / Y$ is a compact complex variety in $\mathcal{C}, \eta$ is a generically smooth 
fiber space with any smooth fiber a complex torus and $\phi$ is a meromorphic $Y$ map (which is necessarily holomorphic over some Zariski open subset of $Y$ ) with the following universal property: Let $\nu: \tilde{Y} \rightarrow Y$ be any proper surjective morphism with $\tilde{Y}$ a variety. Then for any commutative diagram

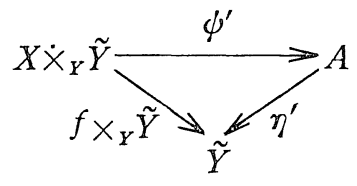

where $\psi^{\prime}$ is a meromorphic $\tilde{Y}$-map, $A$ is a compact complex variety in $\mathcal{C}$ and $\eta^{\prime}$ is a generically smooth fiber space with any smooth fiber a complex torus, there exists a unique meromorphic $Y$-map $b:\left(\mathrm{Alb}^{*} X / Y\right) \dot{\mathrm{X}}_{Y} \tilde{Y} \rightarrow A$ such that $\phi^{\prime}=b\left(\phi \dot{\times}_{Y} \tilde{Y}\right)$. We also call $\phi$ itself a relative Albanese map for $f$. We call Alb* $X / Y$ a relative Albanese variety associated to $f$. Clearly $\mathrm{Alb}^{*} X / Y$ is unique up to bimeromorphic equivalences over $Y$ if one exists.

Theorem 2. Let $f: X \rightarrow Y$ be a generically smooth fiber space of compact complex varieties in $\mathcal{C}$ with $a(f)=\operatorname{dim} f$. Then there exists a relative Albanese map (*) for $f$ with the following additional properties. 1) There exists a Zariski open subset $V \leqq Y$ such that both $X$ and $\mathrm{Alb}^{*} X / Y$ are smooth over $V$ and the induced map $\psi_{V}: X_{V} \rightarrow\left(\mathrm{Alb}^{*} X / Y\right)_{V}$ is holomorphic and isomorphic to the Albanese map for the smooth morphism $f_{V}$, and 2) the map $\psi: X \rightarrow \mathrm{Alb}^{*} X / Y$ is Moishezon (i.e., any of its holomorphic model is Moishezon). Moreover if $f$ is Moishezon, $\eta:$ Alb* $X / Y \rightarrow Y$ also is Moishezon.

Corollary. A meromorphic $Y$-map $\psi^{\prime}: X \rightarrow A$ of $X$ into a compact complex variety $A$ in $C$ over $Y$ is a relative Albanese map for $f$ if there exists a Zariski open subset $U \subseteq Y$ such that for $y \in U, X_{y}, A_{y}$ are smooth and the induced map $\psi_{y}^{\prime}: X_{y} \rightarrow A_{y}$ is holomorphic and isomorphic to an Albanese map of $X$.

Proof. By the universality of $\phi: X \rightarrow \mathrm{Alb}^{*} X / Y$ there exists a unique meromorphic $Y$-map $u: A l b * X / Y \rightarrow A$ such that $u \psi=\psi^{\prime}$. On the other hand, from our assumption it follows that $u$ must give an isomorphism of any fiber over $y \in V$. Hence $u$ is bimeromorphic and $\psi^{\prime}$ is a relative Albanese map. q.e.d.

4.2. Proof of Theorem 2. Let $U \subseteq Y$ be a Zariski open subset over which $f$ is smooth.

I. Construction of $\mathrm{Alb}^{*} X / Y$. a) First we assume that there exists a meromorphic section $s: Y \rightarrow X$. We then set $\mathrm{Alb}^{*} X / Y:=\mathrm{Pic}_{0}^{*}\left(\left(\mathrm{Pic}_{0}^{*} X / Y\right) / Y\right)$. Since $\mathrm{Pic}_{0}^{*} X / Y$ is in $\mathcal{C}$ and generically smooth over $Y$, this makes sense. Note that by the property 7) of $\mathrm{Pic}^{*} X / Y$ Alb* $X / Y$ is Moishezon over $Y$ if $f$ is. Let $\mathcal{L}_{0}$ be a meromorphic relative Poincaré sheaf on $X \dot{\times}_{Y} \mathrm{Pic}_{0}^{*} X / Y$ constructed in Proposition 5 with $W=Y$ there. Let $\phi=\phi_{X}: X \rightarrow \mathrm{Alb}^{*} X / Y$ be the universal mero- 
morphic $Y$-map deifned by $\mathcal{L}_{0}$. (See Definition 4 6) applied to $f: X \rightarrow Y$ and $\mathcal{L}_{0}$ instead of to $\nu: \tilde{Y} \rightarrow Y$ and $\mathscr{I}$ respectively.) We claim that $\phi$ is a desired relative Albanese map for $f$. For any $y \in U_{0}$ (cf. Definition 41 )) $\phi$ induces a map $\phi_{y}$ : $X_{y} \rightarrow \mathrm{Pic}_{0}\left(\mathrm{Pic}_{0} X_{y}\right)$, and this coincides with the Albanese map of $X_{y}$ by the construction of $\psi$ in view of 1.6 a). Hence by Proposition 1 the additional property 1) of $\phi$ in the above proposition is checked. In particular, if the general fiber of $f$ is an abelian variety, $\phi$ is bimeromorphic. We shall next prove the universality of $\phi$. Let $g: A \rightarrow Y$ be any generically smooth fiber space of compact complex varieties in $\mathcal{C}$ whose general fiber is a complex torus. Let $\phi^{\prime}: X \rightarrow A$ be an arbitrary meromorphic $Y$-map, which is necessarily holomorphic over some Zariski open subset of $Y$. (For simplicity of notation we consider only the case $Y=\tilde{Y}$ in Definition 5.)

a1) First we assume that the general fiber of $g$ is an abelian variety, i. e., $a(g)=\operatorname{dim} g$. Then by the property 3$)$ of $\mathrm{Pic}^{*} X / Y, \psi^{\prime}$ induces a meromorphic $Y$-map $\operatorname{Pic}_{0}^{*} A / Y \rightarrow \operatorname{Pic}_{0}^{*} X / Y$ which in turn induces a meromorphic $Y$-map $a: \mathrm{Alb}^{*} X / Y \rightarrow \mathrm{Alb}^{*} A / Y$ again by the property 3$)$ where $\mathrm{Alb}^{*} A / Y$ $=\operatorname{Pic}_{0}^{*}\left(\left(\operatorname{Pic}_{0}^{*} A / Y\right) / Y\right)$ as above. Since $g$ admits the meromorphic section $\psi^{\prime}$ s we

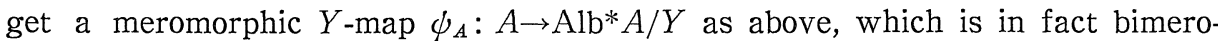
morphic as we have remarked above. Then setting $a^{\prime}=\psi_{A}^{-1} a$, we claim that $\psi^{\prime}=a^{\prime} \phi$. In fact, it is enough to check this on the general fiber of $f$ and hence to check this in the absolute case. And in the absolute case this is true in view of 1.6 a).

a2) It remains to consider the case where $a(g)<\operatorname{dim} g$. In this case, by what we have proved above, it suffices to show that $\psi^{\prime}$ factors through a subvariety $A_{1} \leqq A$ whose general fiber $A_{1, y}$ over $Y$ is an abelian subvariety of $A_{y}$. By Proposition 1, over $U$ we have a natural morphism $a(U):$ Alb $X_{U} / U \rightarrow A_{U}$ such that $\psi_{U}^{\prime}=a(U) \psi_{U}$. Moreover, the image $A_{1}(U):=a(U)\left(\operatorname{Alb} X_{U} / U\right)$ contains $\phi^{\prime} s(U)$ and its fiber $A_{1}(U)_{y}$ over $y \in U$ is an abelian subvariety of $A_{y}$. We show that the closure $A_{1}$ of $A_{1}(U)$ in $A$ is analytic. Let $S^{\prime}=\psi^{\prime} s(Y) \cong A$. Let $D_{A / Y}\left(S^{\prime}\right)$ $=\left\{d \in D_{A / Y, \text { red }} ; Z_{A / Y, d} \ni S_{d}^{\prime}:=\psi^{\prime} s(d)\right\}$ where $D_{A / Y}$ is the relative Douady space for $g$. Then $D_{A / Y}\left(S^{\prime}\right)$ is an analytic subset of $D_{A / Y, \text { red }}$. Let $\tau(U): U \rightarrow D_{A / Y}\left(S^{\prime}\right)_{U}$ be the universal $U$-morphism associated to the inclusion $A_{1}(U) \subseteq A_{U}$. Let $D_{\alpha}$ be the irreducible component of $D_{A / Y}\left(S^{\prime}\right)$ which contains $\tau(U)(U)$. Since, for any $y \in U, D_{\alpha, y}$ contains the point $d(y)$ corresponding to $A_{1}(U)_{y}$ as an isolated point, $\tau(U)(U)$ must be Zariski open in $D_{\alpha}$. This implies that $D_{a, U}=\tau(U)(U)$. Hence the natural image $A_{1}$ of the universal subspace $Z_{\alpha} \subseteq A \times_{Y} D_{\alpha}$ in $A$ is the desired subspace of $A$ which is the closure of $A_{1}(U)$.

b) We consider the general case. Let $\tilde{Y}=X, \tilde{X}=X \dot{\times}_{Y} \tilde{Y}$. Let $\tilde{f}: \tilde{X} \rightarrow \tilde{Y}$ be the natural morphism. We set $\nu=f: \tilde{Y} \rightarrow Y$. Let $\tilde{U}=\nu^{-1}(U)$. Since $\tilde{f}$ admits a holomorphic section, by what we have proved in a) we have the relative Albanese map $\tilde{\psi}: \tilde{X} \rightarrow \mathrm{Alb} * \tilde{X} / \tilde{Y}$ for $\tilde{f}$. On the other hand, by our construction of Alb* $\tilde{X} / \tilde{Y}$ we see readily that if we restrict $U,\left(\operatorname{Alb}^{*} \tilde{X} / \tilde{Y}\right) \tilde{U}$ is smooth over $\tilde{U}$, and 
then, it is isomorphic to $\operatorname{Alb} \tilde{X}_{\tilde{U}} / \tilde{U} \cong\left(\operatorname{Alb} X_{U} / U\right) \times_{U} \tilde{U}$. Let $u:(\operatorname{Alb} * \tilde{X} / \tilde{Y}) \tilde{U}$ $\rightarrow \operatorname{Alb} X_{U} / U$ be the induced morphism. Then $u$ is smooth and hence by Lemma 9 there exists a Zariski open embedding $\operatorname{Alb} X_{U} / U \rightarrow \operatorname{Alb} * X / Y$ with $\mathrm{Alb}^{*} X / Y$ a compact complex variety in $\mathcal{C}$ over $Y$ such that $u$ extends to a meromorphic $Y$-map $u^{*}: \mathrm{Alb}^{*} \tilde{X} / \tilde{Y} \rightarrow \mathrm{Alb}^{*} X / Y$. Then, since $u \tilde{\psi}=\psi_{X_{U}} \tilde{\nu}$ on $\tilde{X}_{\widetilde{U}}$ where $\tilde{\nu}: \tilde{X} \rightarrow X$ is the natural map and $\psi_{X_{U}}: X_{U} \rightarrow \mathrm{Alb} X_{U} / U$ is the relative Albanese map for the smooth morphism $f_{U}$, by Lemma $10 \tilde{\psi}$ induces a meromorphic extension $\psi: X \rightarrow \mathrm{Alb}^{*} X / Y$ of $\psi_{X_{U}}$. The universality can be seen in a similar way by reducing to the absolute case as in a). If $f$ is Moishezon, then $\tilde{f}$, and hence Alb* $\tilde{X} / \tilde{Y} \rightarrow \tilde{Y}$ also, is Moishezon. Hence $\mathrm{Alb}^{*} X / Y \rightarrow Y$ is Moishezon by ([6], Prop. 1).*)

II. Moishezonness of $\psi$. By Proposition 4 passing to another bimeromorphic model we may assume that $f$ is generically locally projective. (By the property 1) and the universality, the relative Albanese map is bimeromorphically invariant.) Take and fix an s. ample $\alpha \in \Gamma(f)$ such that $\operatorname{Pic}_{\alpha}^{*} X / Y \rightarrow Y$ is a fiber space (Lemma 5). Since $\alpha$ is s. ample, $\operatorname{Div}_{\alpha}^{*} X / Y$ is smooth over $U_{\alpha}$, and $\left(\operatorname{Div}_{\alpha}^{*} X / Y\right)_{y}$ is connected for any $y \in Y$. In particular for $y \in U_{\alpha}$, there exists a unique $\alpha_{y} \in N S\left(X_{y}\right)$ such that $\left(\operatorname{Div}_{\alpha}^{*} X / Y\right)_{y}=\operatorname{Div}_{\alpha_{y}} X_{y}$. Let $Z_{\alpha}^{*} \subseteq X \dot{\times}_{Y} \operatorname{Div}_{\alpha}^{*} X / Y$ be the meromorphic universal divisor. Considering $X$ as a parameter space we have the universal meromorphic $Y$-map $\varphi_{\alpha}: X \rightarrow \operatorname{Div}^{*}\left(\left(\operatorname{Div}_{\alpha}^{*} X / Y\right) / Y\right)$. For simplicity write $D_{\alpha}^{*}=$ $\operatorname{Div}_{\alpha}^{*} X / Y$. Let $\operatorname{Pic}_{r}^{*}\left(D_{\alpha}^{*} / Y\right)$ be the unique irreducible component of $\operatorname{Pic}^{*}\left(D_{\alpha}^{*} / Y\right)$ containing the image of $X$ under the composite meromorphic map $\mu_{D_{\alpha}^{*} / Y} \varphi_{\alpha}$ : $X \rightarrow \operatorname{Pic} *\left(D_{\alpha}^{*} / Y\right)$. Let $\phi_{\alpha}: X \rightarrow \operatorname{Pic}_{\gamma}^{*}\left(D_{\alpha}^{*} / Y\right)$ be the induced map.

We claim that $\phi_{\alpha}$ is a relative Albanese map for $f$. For this, it suffices by Corollary (which depends only on the Property 1) of $(*)$ ) to show that for general $y \in U$ the induced morphism $\psi_{\alpha, y}: X_{y} \rightarrow \operatorname{Pic}_{r}^{*}\left(D_{a}^{*} / Y\right)_{y}$ is isomorphic to the Albanese map of $X_{y}$. We first note that $\operatorname{Pic}_{\gamma}^{*}\left(D_{\alpha}^{*} / Y\right)_{y}$ is connected. In fact, let $\beta_{r, 1}^{*}$ : $\mathrm{Pic}_{\gamma}^{*} D_{\alpha}^{*} / Y \rightarrow \bar{P}_{\gamma}^{*}, \beta_{r, 2}^{*}: \bar{P}_{\gamma}^{*} \rightarrow Y$ be the Stein factorization of $\beta_{\gamma}^{*}: \mathrm{Pic}_{\gamma}^{*} D_{\alpha}^{*} / Y \rightarrow Y$. Then $\beta_{r, 1}^{*} \psi_{\alpha}(X) \subseteq \bar{P}_{r}^{*}$ gives a meromorphic section to $\beta_{r, 2}^{*}$ since $f$ is a fiber space. Hence $\beta_{r, 2}^{*}$ is bimeromorphic and $\beta_{\gamma}^{*}$ is a fiber space as was desired. Thus there exists a unique $\gamma_{y} \in N S\left(D_{\alpha, y}^{*}\right)$ such that $\left(\operatorname{Pic}_{\gamma}^{*} D_{a}^{*}\right)_{y}=\operatorname{Pic}_{\gamma_{y}}\left(\operatorname{Div}_{\alpha_{y}} X_{y}\right)$ for $y \in U_{\alpha}$. Moreover $\psi_{\alpha, y}: X_{y} \rightarrow \operatorname{Pic}_{\gamma_{y}}\left(\operatorname{Div}_{\alpha_{y}} X_{y}\right)$ is precisely the morphism defined from the inclusion $Z_{\alpha, y}^{*}=Z_{\alpha y} \subseteq X_{y} \times \operatorname{Div}_{\alpha_{y}} X_{y}$ as in Lemma 2. Hence by that lemma, $\phi_{\alpha, y}$ is an Albanese map of $X_{y}$. Finally since $\alpha$ is s. ample, $X$ is bimeromorphic over $Y$ to the image of $X$ in $\operatorname{Div}_{\gamma}^{*}\left(D_{\alpha}^{*} / Y\right)$ via $\varphi_{\alpha}$, and hence, $\psi_{\alpha}$ is Moishezon since $\mu_{\gamma}^{*}$ is Moishezon by the property 4 ) of $\mathrm{Pic}_{\gamma}^{*} X / Y$.

q. e. d.

4.3. Some applications. Let $g: Z \rightarrow W$ be a fiber space of complex varieties. A meromorphic multi-section to $g$ is an analytic subvariety $B \cong Z$ such that the restriction $\left.g\right|_{B}: B \rightarrow W$ is surjective and generically finite.

*) From our construction it follows that $\mathrm{Alb}^{*} X / Y$ is bimeromorphic over $U$ to Alb $X_{U} / U$ for any $U$ as above. 
Proposition 6. Let $f: X \rightarrow Y$ be a generically smooth fiber space of compact complex varieties in $\mathcal{C}$ with $a(f)=\operatorname{dim} f$. Let $W \leqq Y$ be an open subset. Suppose that there exists a meromorphic multi-section to $f$ defined in a neighborhood $\bar{W}$, the closure of $W$. Then $f_{W}: X_{W} \rightarrow W$ is Moishezon.

Proof. For simplicity of notation we consider only the case $W=Y$. The general case can be treated completely in the same way. Let $B \cong X$ be a meromorphic multisection to $f$. Since $B \rightarrow Y$ is generically finite it is Moishezon. So it suffices to show that $f \times_{Y} B: X \times_{Y} B \rightarrow B$ is Moishezon; we may assume from the beginning that $f$ admits a meromorphic section $s: Y \rightarrow X$. Now by Theorem 2 it suffices to show that $\mathrm{Alb}^{*} X / Y \rightarrow Y$ is Moishezon, so that (considering Alb* $X / Y$ instead of $X$ ) we may assume that the general fiber of $f$ is an abelian variety. Let $U \subseteq Y$ be a Zariski open subset over which $f$ is smooth and on which $s$ is defined. Then there exists on $X_{U}$ a unique structure of a relative complex Lie group over $U$ (cf. [10]). Then by Mumford [20] we can construct a line bundle on $X_{U}$ which is relatively ample with respect to $f_{U}$. Our idea is then nothing but to check that his construction extends 'meromorphically' to the whole $X$. First, by [10] Prop. 7, the relative group multiplication $X_{U} \times_{U} X_{U} \rightarrow X_{U}$ of $X_{U}$ extends to a meromorphic $Y$-map $b^{*}: X \dot{\mathrm{X}}_{Y} X \rightarrow X$. Take an s. ample component $P_{\gamma}^{*}:=\operatorname{Pic}_{\gamma}^{*} X / Y$ which is a fiber space over $Y$ (Lemma 5). Let $\mathcal{L}_{\gamma}$ be a meromorphic relative Poincaré sheaf on $X_{\gamma}:=X \dot{\times}_{Y} P_{\gamma}^{*}$ associated to $f$ and $s$ (Proposition 5). Let $b_{\gamma}^{*}: X_{\gamma} \dot{\times}_{P_{\gamma}^{*}} X_{\gamma} \rightarrow X_{\gamma}$ be induced by $b^{*}$. Let $p_{i}: X_{i} \dot{\times}_{P_{\gamma}^{*}} X_{\gamma}$ $\rightarrow X_{\gamma}$ be the projections to the $i$-th factors. Set $\mathscr{M}_{\gamma}=b_{\gamma}^{*} \mathcal{L}_{\gamma} \otimes p_{1}^{*} \mathcal{L}_{\gamma}^{\sim} \otimes p_{2}^{*} \mathcal{L}_{\gamma}^{r}$ (cf. 3.4 a)) which is a coherent analytic sheaf on $X_{\gamma} \dot{\times}_{P_{\gamma}^{*}} X_{\gamma}$ and is invertible on some Zariski open subset of $Y$. Consider $X_{\gamma} \dot{\otimes}_{P_{\gamma}^{*}} X_{Y}$ as a complex space over $X_{\gamma}$ via $p_{1}$. Then by the property 6$)$ of $\mathrm{Pic}^{*} X / Y, \mathscr{M}_{\gamma}$ defines the universal meromorphic $P_{\gamma}^{*}$ map $X_{\gamma} \rightarrow \operatorname{Pic}_{0}^{*}\left(X_{\gamma} / P_{\gamma}^{*}\right)$, denoted by $\Lambda\left(\mathcal{L}_{\gamma}\right)$, which is holomorphic over $P_{\gamma, U}^{*}:=P_{\gamma}^{*} \times_{Y} U$ (cf. [20], p. 120). Define $\Lambda\left(\mathcal{L}_{\gamma}\right)^{\prime}: X_{\gamma} \rightarrow\left(\operatorname{Pic}_{0}^{*} X / Y\right) \dot{X}_{Y} P_{\gamma}^{*}$ by the composition of $\Lambda\left(\mathcal{L}_{\gamma}\right)$ and the natural bimeromorphic $Y$-map $\operatorname{Pic}_{0}^{*}\left(X_{\gamma} / P_{\gamma}^{*}\right) \rightarrow\left(\operatorname{Pic}_{0}^{*} X / Y\right) \dot{X}_{Y} P_{\gamma}^{*}$ (cf. Def. 4,2)). Then by a theorem of Weil $\Lambda\left(\mathcal{L}_{\gamma}\right)_{U_{0}}^{\prime}$ descends to a $U$-morphism ${\overline{\Lambda\left(\mathcal{L}_{\gamma}\right)_{U_{0}}}}: X_{U_{0}} \rightarrow \mathrm{Pic}_{0}^{*} X_{U_{0}} / U_{0}$ (cf. [20], p. 120, Def. 6.2), where $U_{0}$ is as in 1) of Defini-

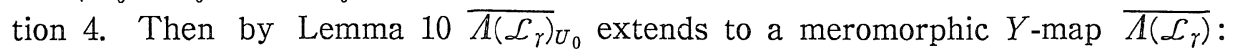
$X \rightarrow \operatorname{Pic}_{0}^{*} X / Y$. We set $\mathscr{I}=j^{*}\left(i d_{X} \times_{Y} \overline{\Lambda\left(\mathcal{L}_{Y}\right)}\right) * \mathcal{L}_{0}$ where $\mathcal{L}_{0}$ is the meromorphic relative Poincaré sheaf on $X \dot{\times}_{Y} \mathrm{Pic}_{0}^{*} X / Y$ and $j: X \rightarrow X \times_{Y} X$ is the embedding as the diagonal. Then by [20] Prop. 6.10, the restriction $\Psi_{y}$ of $\Psi_{1}$ to $X_{y}, y \in U_{0}$, is an ample invertible sheaf. It follows that $f$ is Moishezon. q.e.d.

Proposition 7. Let $f: X \rightarrow Y$ be a generically smooth fiber space of compact complex varieties in $\mathcal{C}$ with $a(f)=\operatorname{dim} f$. Suppose that $q\left(X_{y}\right)=0$ for a general fiber $X_{y}$ of $f$ where $q\left(X_{y}\right):=\operatorname{dim} H^{1}\left(X_{y}, \mathcal{O}_{X_{y}}\right)$ is the irregularity of $X_{y}$. Then $f$ is Moishezon.

Proof. Since $q\left(X_{y}\right)=0$, Alb $^{*} X / Y \rightarrow Y$ is bimeromorphic. Hence $f$ is bimero- 
morphic to its Albanese map $\phi: X \rightarrow \mathrm{Alb}^{*} X / Y$ which is Moishezon by Theorem 2. q. e. d.

A proper morphism $f: X \rightarrow Y$ of complex spaces is called locally Moishezon if for any $y \in Y$ there exists a neighborhood $y \in V$ such that $f_{V}: X_{V} \rightarrow V$ is Moishezon. By Chow lemma [15] it is immediate to see that if $f$ is locally Moishezon, every fiber of $f$ is Moishezon.

Proposition 8. Let $f: X \rightarrow Y$ be a generically smooth fiber space of compact complex varieties in $\mathcal{C}$. Let $U \subseteq Y$ be a Zariski open subset over which $f$ is smooth. Then the following conditions are equivalent. 1) $a(f)=\operatorname{dim} f$, 2) $f_{U}$ : $X_{U} \rightarrow U$ is locally Moishezon, and 3) there exists a bimeromorphic model $f^{*}: X^{*} \rightarrow Y^{*}$ of $f$ which is locally Moishezon.

Proof. By the remark preceding the proposition it is clear the 2) or 3) implies 1). So we show that 1) implies 2) and 3). 1) $\rightarrow 2$ ): Since $\eta_{U}: \operatorname{Alb} X_{U} / U$ $\rightarrow U$ is smooth, we can get a holomorphic section to $\eta_{U}$ at any point of $U$. Since $\mathrm{Alb}^{*} X / Y$ is bimeromorphic over $U$ to $\mathrm{Alb} X_{U} / U$, $\mathrm{Alb} * X / Y$ then admits a meromorphic section locally at any point of $U$. Hence by Proposition $6 f$ is locally Moishezon. 1) $\rightarrow 3$ ): Let $p: \tilde{Y} \rightarrow Y$ be a proper modification such that the strict transform $\left(\mathrm{Alb}^{*} X / Y\right)^{\sim}$ in $\left(\mathrm{Alb}^{*} X / Y\right) \times_{Y} \tilde{Y}$ is flat over $\tilde{Y}$. Since $(\mathrm{Alb} * X / Y)^{\sim}$ is bimeromorphic to $\operatorname{Alb}^{*}\left(X \times_{Y} \tilde{Y} / \tilde{Y}\right)$, it follows that $\operatorname{Alb}^{*}\left(X \times_{Y} \tilde{Y} / Y\right)$ admits a meromorphic multi-section at any point of $\tilde{Y}$. Hence $f_{\tilde{Y}}: X \times_{Y} \tilde{Y} \rightarrow \tilde{Y}$ is locally Moishezon by Proposition 6 . Take $f^{*}=f_{\tilde{Y}}$.

q. e. d.

Remark 2. In general even if $a(f)=\operatorname{dim} f, f$ may not be locally Moishezon unless we take a flattening of $f$. In fact, let $f: X \rightarrow S$ be a flat elliptic fiber space such that $f$ is an algebraic reduction of $X$, where $\operatorname{dim} X=3$ and $\operatorname{dim} S=2$. Suppose that there exists an irreducible exceptional curve of the first kind $C$ on $S$ such that $f: X_{C} \rightarrow C$ is an algebraic reduction of $X_{C}$. Let $\phi: S \rightarrow S^{\prime}$ be the contraction of $C$ to a smooth point $p \in S^{\prime}$. Then $a\left(f^{\prime}\right)=\operatorname{dim} f^{\prime}=1$ for $f^{\prime}=\phi f$ : $X \rightarrow S^{\prime}$ while $f^{-1}(p)=X_{C}$ is not Moishezon. Further it is easy to find an actual example of such.

Proposition 9. Let $f: X \rightarrow Y$ be a generically smooth fiber space of compact complex varieties in $\mathcal{C}$. Suppose that $\operatorname{dim} X=3$. Then the relative Albanese map for $f$ exists except possibly the case where the general fiber of $f$ is an elliptic surface with trivial homological invariant (cf. [18]).

Proof. If $a(f)=\operatorname{dim} f$, this follows from Theorem 2. If $\operatorname{dim} f=0$ or 3 , then the proposition is clearly true. So we may assume that $\operatorname{dim} f=2$.

If $a(f)=1$, then the general fiber of $f$ is an elliptic surface. Let $\phi(U): X_{U}$ $\rightarrow$ Alb $X_{U} / U$ be the relative Albanese map for $f_{U}$ where $U$ is a Zariski open subset of $Y$ over which $f$ is smooth. Let $\phi(U)\left(X_{U}\right)=C(U)$. Suppose that $X_{y}$ has non-trivial homological invariant. Then we have $\operatorname{dim} C(U)=2$ and the induced 
map $\varphi(U): X_{U} \rightarrow C(U)$ is a flat fiber space (cf. [18]). Hence by Lemma 9 there exists a Zariski open embedding $C(U) \subseteq C$ with $C$ a compact complex variety in $C$ over $Y$ such that $\varphi(U)$ extends to a meromorphic $Y$-map $\varphi: X \rightarrow C$. Since $b: C \rightarrow Y$ has relative dimension 1 and hence $a(b)=1$, by Theorem 2 we have the relative Albanese map $\phi_{C}: C \rightarrow \mathrm{Alb}^{*} C / Y$ for $b$. Then it is immediate to see that $\psi_{C} \varphi: X \rightarrow \mathrm{Alb} * C / Y$ is the desired Albanese map for $X$ (cf. Corollary to Theorem 2).

Finally suppose that $a(f)=0$. Then $X_{y}$ is either bimeromorphic to a complex torus or a K3 surface. In the latter case there is nothing to prove since $q\left(X_{y}\right)=0$. In the former case we use [11] $\S 1$ Theorem; according to it either $X_{y}$ is isomorphic to a complex torus or $f$ is bimeromorphic to a morphism $(S \times E) / G \rightarrow E / G$ where $E$ is a compact Riemann surface, $S$ is a complex torus, and $G$ is a finite group acting on both $E$ and $T$. In the first case we may set $X=\mathrm{Alb}^{*} X / Y$, and in the second case we can take $(S \times E) / G$ as the relative Albanese variety by Corollary to Theorem 2 .

q. e. d.

Final Remark. Let $f: X \rightarrow Y$ be a fiber space of compact complex varieties. We say that $f \in \mathcal{C} / Y$ if there exist a proper locally Kähler morphism $g: Z \rightarrow Y$ and a surjective meromorphic $Y$-map $\varphi: Z \rightarrow X$ (cf. [6]). Then the results of this paper are true even if the condition $X \in \mathcal{C}$ is replaced by a weaker one $f \in \mathcal{C} / Y$ if in the statements everything is restricted to an arbitrary relatively compact open subset of $Y$. (In particular if $Y$ is compact no restriction is needed.)

\section{References}

[1] Banica, C. and Stanasila, C., Algebraic methods in the global theory of complex spaces, John Wiley Sons and Editura Academiei, 1976.

[2] Bingener, J., Darstellbarkeitskriterien für analytische Funktoren, Ann. Sci. Ecole Norm. Sup., 13 (1980), 317-347.

[3] Campana, F., Réduction algébrique d'un morphisme faiblement Kählérien propre et applications, Math. Ann., 256 (1981), 157-189.

[4] Fischer, G., Complex analytic geometry, Lecture Notes in Math., 538 Springer, 1976.

[5] Fujiki, A., Closedness of the Douady spaces of compact Kähler spaces, Publ. RIMS, Kyoto Univ., 14 (1978), 1-52.

[6] - On the Douady space of a compact complex space in the category $\mathcal{C}$, Nagoya J. Math., 85 (1982), 189-211.

[7] - A Theorem on bimeromorphic maps on compact Kähler manifolds, Publ. RIMS, Kyoto Univ., 17 (1981), 735-754.

[8] - Some results in the classification theory of compact complex manifolds in $\mathcal{C}$, Proc. Japan Acad. 56 (1980), 324-327.

[8a] — On the Douady space of a Kähler space, Application to the classification theory of compact complex manifolds in $\mathcal{C}$, Report of a conference at Nagoya, 1980, 349-394 (In Japanese).

[9] - Projectivity of the space of divisors on a normal compact complex space, Publ. RIMS, Kyoto Univ., 18 (1982), 1163-1173. 
[10] - On a holomorphic fiber bundle with meromorphic structure, Publ. RIMS, Kyoto Univ., 19 (1983), 117-134.

[11] - On the structure of compact complex manifolds in $\mathcal{C}$, Advanced Studies in Math., 1, ed. S. Iitaka and H. Morikawa, (1982), 229-300.

[12] — Relative Albanese map for a proper smooth morphism and its applications, in preparation.

[13] Grothendieck, A., Fondements de la géométrie algebrique (Extraits du Seminaire Bourbaki 1957-1962), Paris 1962.

[14] — Technique de construction en géométrie analytique, Séminaire H. Cartan, 13e année, 1960-61.

[15] Hironaka, H., Flattening theorem in complex analytic geometry, Amer. J. Math., 96 (1975), 503-547.

[16] Hirshowitz, A., Sur les plongements du type deformation, Comment. Math. Helvetici, 54 (1979), 126-132.

[17] Kodaira, K., On Kähler varieties of restricted type, Ann. of Math., 60 (1954), $28-48$.

[18] - On compact analytic surfaces, II and III, Ann. of Math., 77 (1963), 563-626 and 78 (1963), 1-40.

[19] Lieberman, D. and Sernesi, E., Semi-continuity of L-dimension, Math. Ann., 225 (1977), 77-88.

[20] Mumford, D., Geometric invariant theory, Berlin-Heidelberg-New-York: Springer 1965.

[21] Rossi, H., Picard varieties of an isolated singular point, Rice Univ. Studies, 54 (1968), 63-73.

[22] Schuster, H.W., Zur Theorie der Deformationen kompakter komplexer Räume, Inventiones math., 9 (1970), 284-294.

[23] Ueno, K., Classification theory of algebraic varieties and compact complex spaces, Lecture Notes in Math., 439, Springer 1975.

Note added in Proof. The relative Albanese map with the property 1) of Theorem 2 has recently been constructed by $\mathrm{F}$. Campana without the assumption that $\operatorname{dim} f=a(f)$, by quite a different method. 